\title{
ESTUDIO FLORÍSTICO EN EL ÁREA DE LA COMUNIDAD INDÍGENA DE NUEVO SAN JUAN PARANGARICUTIRO, MICHOACÁN, MÉXICO ${ }^{1,2}$
}

\author{
Consuelo Medina García, Fernando Guevara-Féfer \\ Marco Antonio Martínez Rodríguez, Patricia Silva-Sáenz, \\ Ma. Alma Chávez-Carbajal \\ Facultad de Biología \\ Universidad Michoacana de San Nicolás de Hidalgo \\ 58060 Morelia, Michoacán \\ E \\ IgnaCio García RuIZ ${ }^{3}$ \\ CIIDIR - IPN Michoacán \\ Justo Sierra 28 \\ 59510 Jiquilpan, Michoacán
}

\section{RESUMEN}

El estudio florístico realizado en el área de la comunidad indígena de Nuevo San Juan Parangaricutiro registró la presencia de 108 familias, con 307 géneros, 716 especies y 16 taxa infraespecíficos, de los cuales, 52 son helechos y afines, 16 gimnospermas, 120 monocotiledóneas y 544 dicotiledóneas. Las familias mejor representadas son: Compositae (135), Leguminosae (58), Gramineae (57), Labiatae (26), Solanaceae (21) Orchidaceae (20) y Polypodiaceae (18). 60.7\% de las especies corresponden a la forma de vida herbácea (perenne y anual), $19.1 \%$ son arbustos, $10.0 \%$ árboles, $4.2 \%$ trepadoras, $3.3 \%$ epífitas, $1.8 \%$ parásitas, "saprófitas" $0.5 \%$ y acuáticas $0.4 \%$.

\section{ABSTRACT}

The inventory of the vascular flora in the area of comunidad indígena de Nuevo San Juan Parangaricutiro produced the following results: 108 families with 307 genera, 716 species and 16 infraespecific taxa. From this total 52 species belong to pteridophytes, 16 to gymnosperms, 120 to monocotyledons and 544 to dicotyledons. The best represented families, in terms of species number are: Compositae (135), Leguminosae (58), Gramineae (57), Labiatae (26), Solanaceae (21), Orchidaceae (20), and Polypodiaceae (18). 60.7\% of the species are herbaceous (either perennial or annual plants); $19.1 \%$ are shrubs, $10.0 \%$ trees, $4.2 \%$ lianas, $3.3 \%$ are epiphytic plants, $1.8 \%$ are parasites, "saprophytes" amount to $0.5 \%$ and aquatics $0.4 \%$.

\footnotetext{
1 Trabajo realizado con apoyo económico de la Universidad Nacional Autónoma de México. Programa DGAPA (IN209094).

2 Con apoyo económico de la Comisión Nacional para el Conocimiento y Uso de la Biodiversidad. Proyecto H-304.

${ }^{3}$ Becario de la Comisión de Operación y Fomento de Actividades Académicas del I.P.N. (COFAA).
} 


\section{INTRODUCCIÓN}

Este trabajo es parte de la investigación que realiza la Universidad Nacional Autónoma de México a solicitud de los habitantes de la comunidad indígena de Nuevo San Juan Parangaricutiro, quienes están interesados en fortalecer el plan de manejo de sus recursos naturales. El proyecto pretende generar un mejor conocimiento de las plantas que crecen en su territorio y poder así, proteger y utilizar esta gran cantidad de vegetales. Los integrantes de la comunidad han comprendido el verdadero valor de sus bosques y actualmente, mediante un manejo silvícola apropiado, logran obtener múltiples beneficios.

El presente estudio tuvo como objetivo principal la elaboración de un listado florístico de la flora vascular que se encuentra en la región. Se han incluido datos ecológicos, como altitud, forma de vida y el tipo de vegetación en donde se han colectado los ejemplares.

\section{ANTECEDENTES}

En la época colonial el área de estudio formaba parte de la municipalidad de Uruapan (Moheno,1985); la comarca fue visitada en su viaje a Michoacán por Francisco Hernández, protomédico de las Indias, informando de 15 plantas originarias de esta región (Hernández, 1959). En 1790 recorrieron la zona Sessé y Mociño, y posteriormente Humboldt y Bonpland en 1803 (Bello y Madrigal, 1996).

Trabajos realizados muy cerca y anteriores a la erupción del volcán Paricutín fueron los de Hoogstrall (1941) y Leavenworth (1946), quienes colectaron 1600 ejemplares y describieron la vegetación a lo largo del transecto Río Tepalcatepec-Tancítaro.

Después del nacimiento del Paricutín, Eggler (1948, 1959, 1963), Beaman (1960), Rejmánek et al. (1982) y Giménez de Azcárate et al. (1997) realizaron estudios sobre los avances de la vegetación en las áreas cubiertas por lava y cenizas.

Investigaciones botánicas recientes que abarcaron la región de nuestro interés son las de Madrigal (1982), Bello (1984, 1985, 1993), Bello y Labat (1987), Delgado (1992), Rodríguez y Espinosa (1995, 1996), Espinosa y Rodríguez (1995, 1996). Un trabajo preliminar de la flora y vegetación en la zona de estudio fue el realizado por Martínez (1997), en el que registró 286 especies y reconoció ocho asociaciones vegetales.

\section{CARACTERIZACIÓN DEL ÁREA}

\section{Localización}

El área de la comunidad indígena de Nuevo San Juan Parangaricutiro se localiza en la zona centro-oeste del estado de Michoacán, en el municipio de Nuevo Parangaricutiro. Está situada entre los paralelos $19^{\circ} 21^{\prime}$ y $19^{\circ} 34^{\prime}$ de latitud norte y de los meridianos $102^{\circ}$ 08' y $102^{\circ} 17^{\prime}$ de longitud oeste de Greenwich. Ocupa una superficie de 18318 ha (Martínez, 1997) y tiene forma irregular con longitud axial de cerca de $25 \mathrm{~km}$ y su parte más ancha de más o menos 12 km (Fig. 1). 
Medina et al.: Estudio Florístico de Nuevo San Juan Parangaricutiro, Michoacán, México

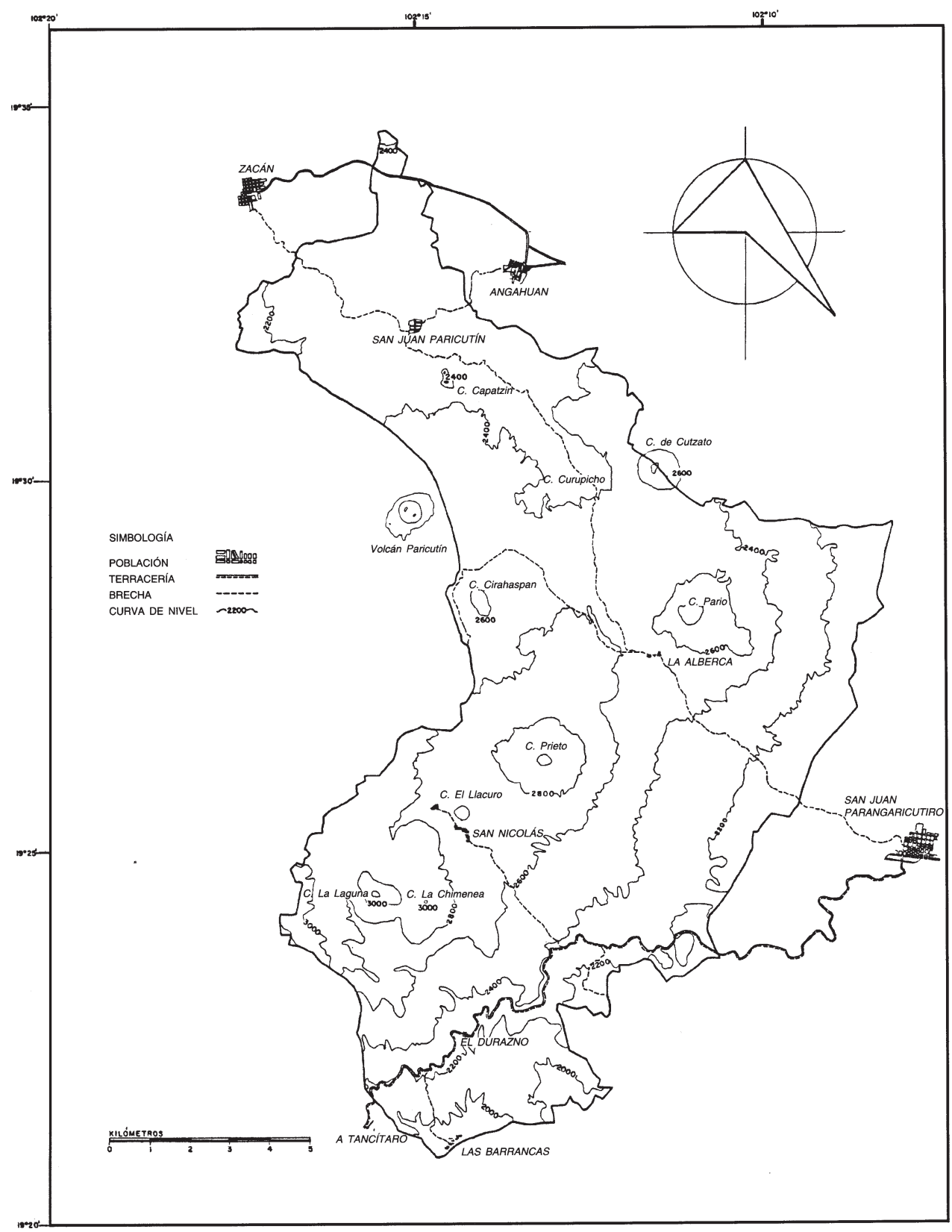

Fig. 1. Área de la comunidad indígena de Nuevo San Juan Parangaricutiro, Michoacán, México. 
Esta zona forma parte del Eje Volcánico Transversal, dentro de la región denominada Meseta Tarasca (Anónimo, 1981). Su límite meridional está en contacto con la transición fisiográfica a la depresión del Balsas (Bocco et al., 1998).

Orografía

Topográficamente la zona de estudio es un terreno accidentado con pendientes de $5 \%$ a $>30 \%$. Las elevaciones más altas se encuentran hacia el centro-sur del área de oeste a este y corresponden a los cerros: La Laguna con 3200 m s.n.m. y La Chimenea con $3000 \mathrm{~m}$, ambos prolongación del cerro Tancítaro (la mayor eminencia del estado con casi 4000 m s.n.m.), el cerro Prieto con 3040 m, el cerro Pario 2910 m y el cerro de San Nicolás $2900 \mathrm{~m}$. Las elevaciones secundarias presentan alturas que varían de 2800 a $2500 \mathrm{~m}$ s.n.m. distribuidas principalmente en el norte; las partes más bajas se sitúan al sur con una altitud de 1800 a $1900 \mathrm{~m}$. Las barrancas nacen en las laderas del Tancítaro y se localizan en la zona sur del territorio comunal (Anónimo, 1987, 1990; Bocco et al., 1998).

Geología

El área de estudio está situada en el Eje Volcánico Transversal que es de las cadenas montañosas más jóvenes del país y su origen data de los periodos Plioceno, Pleistoceno, Holoceno (Bocco et al., 1998) y Reciente. Actualmente, $50 \%$ de los terrenos se presentan cubiertos por espesores variables de cenizas o de malpaíses, resultado de las emisiones del volcán Paricutín, localizado a pocos kilómetros del lindero occidental, que estuvo en actividad a mediados del siglo XX. Las principales rocas son ígneas extrusivas del tipo basáltico y andesítico, así como sus correspondientes tobas.

Suelos

Bocco et al. (1998), identificaron cuatro tipos de suelo: andosol mólico y háplico; regosol en sus subdivisiones vítrico y éutrico sobre andosoles; los leptosoles líticos se encontraron sobre derrames lávicos del Paricutín, y leptosoles andi-mólicos y líticos, asociados con andosoles mólicos y por último los fluvisoles vítrico y éutrico sobre planicies acumulativas.

Hidrografía

La zona estudiada se sitúa en la Región Hidrológica del Balsas (Anónimo, 1981) dentro de tres subcuencas: al oeste la subcuenca del Río Itzícuaro, al este la del Río Cupatitzio y al sur la subcuenca del Río Tepalcatepec.

Atraviesan el área una serie de escurrimientos que forman corrientes durante todo el año, originados en las laderas del estratovolcán El Tancítaro; entre los más importantes se encuentran los que forman las barrancas Agua Chiquita, La Pedregosa y La Culebra, que recorren el territorio comunal, del oeste al sur. 
Martínez (1997), mencionó la presencia de 10 manantiales entre permanentes y temporales, un pequeño cuerpo de agua denominado Los Conejos y un río de nombre Tíscato (Anónimo, 1981).

Clima

No se cuenta con información detallada y completa del comportamiento climático de la región. Con base en los datos de temperatura media y precipitación mensual correspondientes a los años 1989, 1990 y 1991 que proporcionó la estación agrometereológica El Durazno (situada hacia el límite sur de la región de estudio a una altitud de $2300 \mathrm{~m}$ (Martínez, 1997)), el clima es templado húmedo con abundantes lluvias en verano.

La temperatura promedio mensual anual es de $15.1^{\circ} \mathrm{C}$; la mínima se presenta en el mes de diciembre y la máxima en el mes de mayo. La precipitación total anual fue en promedio de $1496.6 \mathrm{~mm}$, siendo el mes más seco marzo (sin registro de precipitación) y julio el mes con mayor cantidad de lluvia $(351.5 \mathrm{~mm})$. 88\% de precipitación se distribuyó durante los meses de junio a octubre (Cuadro 1).

Cuadro 1. Datos de temperatura media y precipitación mensual de la estación El Durazno, Michoacán.

\begin{tabular}{|c|c|c|c|c|c|c|c|c|c|c|c|c|c|}
\hline Meses & $E$ & $\mathrm{~F}$ & $M$ & $A$ & $M$ & J & $\mathrm{J}$ & A & $S$ & O & $\mathrm{N}$ & $\mathrm{D}$ & \\
\hline Temp. ${ }^{\circ} \mathrm{C}$ & 14.0 & 14.3 & 16.1 & 17.2 & 17.3 & 16.1 & 15.0 & 15.2 & 14.9 & 14.7 & 13.8 & 12.7 & Prom. \\
\hline Prec. mm & 10.6 & 4.6 & 0.0 & 32.1 & 47.9 & 191.1 & 351.5 & 278.4 & 317.7 & 189.1 & 40.0 & 33.6 & Total 1496.6 \\
\hline
\end{tabular}

\section{METODOLOGÍA}

La elaboración del inventario florístico se basó en la consulta bibliográfica y principalmente en la determinación de aproximadamente 2500 especímenes que se colectaron en los recorridos por toda el área de la comunidad entre los años 1995, 1996 y 1997. Con el fin de obtener un censo lo más completo posible, se consideraron también aquellas especies que fueron registradas para el área en el trabajo previo a éste por uno de los autores (Martínez, 1997), así como algunos ejemplares incluidos en el listado florístico del estado de Michoacán (Rodríguez y Espinosa, 1995, 1996; Espinosa y Rodríguez, 1995, 1996).

Ejemplares de respaldo de este trabajo serán depositados en los herbarios de la Universidad Michoacana (EBUM), del Centro Regional del Bajío del Instituto de Ecología, A.C. (IEB) y del Centro Interdisciplinario de Investigación para el Desarrollo Integral Regional, Unidad Michoacán (CIMI). 


\section{RESULTADOS}

Vegetación

De acuerdo con la clasificación de Rzedowski y McVaugh (1966) y Rzedowski (1978), en el área de estudio se reconocieron los siguientes tipos principales de vegetación: bosque de coníferas (que incluye: bosque de pino, bosque de oyamel, bosque de oyamel-pino y bosque de pino-oyamel), bosque de pino-encino, bosque mesófilo de montaña, bosque de encino, pastizal y una pequeña comunidad de vegetación subacuática, además de la litoserie y de algunas asociaciones de vegetación secundaria.

Bosques de coníferas. Para la zona este tipo de vegetación lo constituyen comunidades en donde predominan los pinos y los oyameles 0 , lo que es más frecuente, bosques mixtos con intervención de diversas especies de árboles.

Bosque de pino. Los pinares se presentan en suelos de tipo andosol de origen volcánico, los que se caracterizan por ser profundos y con buen drenaje y se establecen en laderas menos protegidas de la insolación. Su área de distribución comprende los cerros Capatzin, Choritiro, Cutzato, Juritzícuaro, cerro Prieto y el cerro Pario, entre otros lugares, en donde por lo general tienen un manejo silvícola. El bosque es siempre verde y presenta troncos rectos de 20 a $30 \mathrm{~m}$ o mayor altura, cuando es más o menos cerrado; son comunidades sombrías, con un sotobosque pobre en especies, por lo general con Baccharis heterophylla, Senecio spp. y Eupatorium spp.

Cuando el bosque está muy perturbado y es muy abierto, como en las laderas del cerro Pario, se han establecido asociaciones densas de Lupinus spp. y Senecio spp. o Satureja macrostema. En cambio, en el cerro Capatzin encontramos Coriaria ruscifolia ssp. microphylla en el estrato arbustivo.

La especie de pino más ampliamente distribuida en el área de estudio es Pinus pseudostrobus que se encuentra desde 1900 hasta 3100 m s.n.m., frecuentemente asociada con $P$. leiophylla, y en menor proporción con $P$. montezumae y $P$. douglasiana. Otros árboles asociados son Crataegus pubescens, Quercus rugosa, Q. laurina, Cleyera integrifolia, Ternstroemia pringlei, Alnus jorullensis ssp. lutea, Oreopanax xalapensis y Salix paradoxa.

Bosque de oyamel. Al igual que en otras partes de México, se distribuye en forma de manchones aislados muchas veces restringido a un cerro, a una ladera o una cañada (Rzedowski, 1978). Las condiciones climáticas que estos bosques requieren para su desarrollo son una precipitación superior a los $1000 \mathrm{~mm}$ y de una temperatura media anual de 7 a $15^{\circ} \mathrm{C}$.

Esta formación se encontró en sitios de alta montaña como en el cerro La Laguna y en laderas del cerro Prieto, o en lugares protegidos en las cañadas del área donde los oyameles forman comunidades en las que son dominantes. Los árboles de Abies religiosa var. religiosa y Abies religiosa var. emarginata son elementos que alcanzan alturas hasta de $40 \mathrm{~m}$ y se asocian con Quercus laurina, Q. rugosa y Pinus pseudostrobus.

En algunos sitios en donde la perturbación del bosque de oyamel es fuerte, como en el cerro La Laguna, se han establecido asociaciones con Alnus jorullensis ssp. jorullensis. 
Bosque de pino-oyamel y oyamel-pino. Estas comunidades se encontraron principalmente en el cerro de La Chimenea, en el cerro La Laguna, en las laderas de la cañada La Culebra y en el cerro Prieto, lugares donde los dos géneros son codominantes, o bien prevalece uno u otro.

Bosque de pino-encino. Eggler (1948, citado por Rzedowski, 1978) cree que los pinares puros de la región de Uruapan, Michoacán, representan una etapa subclímax y que la comunidad madura corresponde al bosque mixto de Pinus y Quercus.

Este tipo de vegetación se encuentra en laderas de los cerros Cuiritzarán, Zacán, Curupicho, Cutzato y Capatzin, en altitudes que van de 2300 hasta 2650 m s.n.m. Comparte los mismos requerimientos ecológicos con el bosque de pino.

Los pinos más frecuentemente asociados a este bosque son: Pinus pseudostrobus, $P$. leiophylla, $P$. douglasiana y $P$. montezumae, acompañados por diversas especies de encinos como Quercus rugosa, Q. martinezii, Q. laurina, Q. crassifolia, Q. obtusata, y en menor proporción con Quercus dysophylla, $Q$. candicans y $Q$. crassipes.

Otros elementos arbóreos comunes son: Alnus jorullensis ssp. lutea, Cleyera integrifolia, Ternstroemia pringlei y Crataegus pubescens. Los géneros de arbustos que se presentan son: Baccharis, Eupatorium, Senecio, Desmodium, Arctostaphylos, Coriaria, Cestrum, Lupinus, Ceanothus, Salvia, Satureja y Trisetum. El estrato herbáceo es rico en especies, sobre todo de las familias de compuestas, gramíneas y leguminosas, así como de numerosos helechos.

Bosque de encino. Se encontró en una área muy reducida en el cerro de San Nicolás, a una altitud de $2750 \mathrm{~m}$ en condiciones de gran disturbio; las especies que componen esta comunidad vegetal son: Quercus conspersa, Q. laurina, asociadas con Alnus jorullensis ssp. lutea y un estrato arbustivo con Senecio angulifolius y Cestrum anagyris.

Bosque mesófilo de montaña. Se localizó en altitudes de 1900 a 2600 m con diferentes grados de conservación a lo largo de las cañadas. Se encuentra mejor representado cerca de El Durazno, a 2300 m, en donde se mezclan árboles con alturas de 20 a 30 m de las especies: Abies religiosa, Pinus pseudostrobus, Quercus laurina, Alnus acuminata ssp. arguta, Oreopanax xalapensis y Prunus brachybotrya. En un segundo dosel se encuentra Cleyera integrifolia, Viburnum elatum, Urera caracasana, Ternstroemia pringlei; en el estrato arbustivo abundan: Salvia mexicana, Psychotria sp., Senecio angulifolius, Viburnum acutifolium ssp. microphyllum, Piper sp., Leandra sp. y Solanum spp. El estrato herbáceo lo conforman Cuphea bustamanta, Phenax hirtus, Smilax moranensis, Eupatorium pazcuarense, y una gran cantidad de helechos como Asplenium monanthes, A. praemorsum y Adiantum poiretii. Existe un estrato rasante con Arenaria lanuginosa y Stellaria cuspidata como elementos principales.

En las mismas cañadas, pero al sur de la región, entre 1900 y 2100 m, se presenta un bosque mesófilo más abierto, en donde se observaron entre otras especies arbóreas a Trema micrantha, Quercus candicans, Alnus acuminata ssp. glabrata, Carpinus caroliniana, Tilia mexicana, Saurauia serrata, Oreopanax echinops y Hedyosmum mexicanum, con abundantes arbustos como Rapanea jurgensenii, Parathesis spp., Salvia albo-caerulea, Fuchsia arborescens, Malvaviscus arboreus, Phymosia rosea, Dendropanax arboreus, y algunas trepadoras como Ipomoea funis, Vitis bourgaeana y Passiflora spp. 
Pastizal. Esta comunidad vegetal se localiza de manera natural en la cima de algunas montañas altas como en el cerro La Laguna (3 200 m), en donde predominan gramíneas amacolladas como Muhlenbergia macroura y Festuca amplissima, alternando con Alchemilla procumbens y Eupatorium pazcuarense. En los llanos de San Nicolás (2 $800 \mathrm{~m}$ ), abundan Muhlenbergia macroura, Aegopogon cenchroides y Acaena elongata.

Vegetación subacuática. En el cerro La Laguna (2 900 m) existe un pequeño cuerpo de agua semipermanente en donde se colectó Potamogeton diversifolius, Heteranthera limosa y Rorippa sp.

Vegetación secundaria. Se incluye en esta categoría a las comunidades de plantas que se establecen como consecuencia de disturbio y que se reconocieron en el campo como arvenses, ruderales, matorral secundario, pastizal inducido y áreas reforestadas. Se describe a continuación las que se consideran de mayor interés.

Litoserie. Con tal término se engloba la vegetación que se ha establecido en las áreas cubiertas por lava procedente de la erupción del volcán Paricutín. En este ambiente se observó un estrato arbóreo muy disperso y de poca altura con Pinus montezumae, $P$. leiophylla, Buddleia cordata, Clethra mexicana, miembros de los bosques establecidos en áreas cercanas. Entre los arbustos se encontró a Baccharis heterophylla, Senecio salignus, Coriaria ruscifolia, Gaultheria cordata ssp. lancifolia y Arctostaphylos discolor. Las herbáceas que se observaron como más frecuentes fueron: Eupatorium pazcuarense, E. glabratum, Aegopogon cenchroides y una gran cantidad de helechos de los géneros Asplenium, Cheilanthes, Pleopeltis, Phlebodium, Polypodium, Pellaea y Elaphoglossum, entre otros.

El estudio de la estructura y composición de las asociaciones que se han establecido en los derrames lávicos del volcán Paricutín ha sido publicado recientemente por Giménez de Azcárate et al. (1997).

Las áreas dedicadas al pastoreo se distribuyen en forma de manchones intercalados con los bosques de pino-encino en terrenos planos o poco inclinados con drenaje deficiente, en donde se establece una asociación con predominio de gramíneas y otras plantas. Dentro de las primeras destaca Muhlenbergia spp., Vulpia myuros, y Setaria geniculata, otras herbáceas son Phacelia platycarpa, Jaegeria hirta, Physalis volubilis, Tagetes filifolia, Oenothera pubescens, y Geranium seemannii.

Entre las especies arvenses asociadas a las huertas de aguacate (2 150 y $2300 \mathrm{~m}$ s.n.m.) destacaron Phacelia platycarpa, Solanum nigrescens, Geranium seemannii, Oxalis corniculata, Sporobolus indicus, Rumex obtusifolius, Bidens odorata y Lepidium virginicum. En terrenos de cultivo abandonado a $2950 \mathrm{~m}$ se encontró Lupinus sp., Romanschulzia arabiformis y especies de los géneros Muhlenbergia, Bromus, Gnaphalium, Conyza, Oenothera, Alchemilla, Sigesbeckia, Oxalis, Jaegeria y Gaura.

\section{LISTADO FLORÍSTICO}

El listado florístico consta de un total de 716 especies de plantas, que corresponden a 108 familias y 307 géneros, además de 16 unidades infraespecíficas. De este total, 593 
especies y 15 unidades infraespecíficas correspondieron al resultado del trabajo de campo de este estudio; en tanto que 111 especies y una variedad fueron colectadas por Martínez (1997) y 12 especies restantes proceden del inventario florístico del estado de Michoacán (ver Apéndice).

La información obtenida se concentró en un listado que se presenta a cuatro columnas, en donde, la primera corresponde a las especies en orden alfabético dentro de cada familia; los nombres que van acompañados de un asterisco corresponden a registros previos a este estudio. La segunda columna indica el tipo de vegetación o la asociación donde fueron encontrados los especímenes. La tercera columna señala los intervalos de altitudes en que fueron colectadas las especies, las que se ajustaron a una cantidad cerrada de $50 \mathrm{~m}$. La cuarta columna atañe a la forma de vida de la planta.

\section{COMPOSICIÓN NUMÉRICA DE LA FLORA}

En el cuadro 2 se reseña la composición taxonómica de la flora vascular en el área de la comunidad indígena de Nuevo San Juan Parangaricutiro y muestra cifras totales de las familias, géneros, especies y unidades infraespecíficas, así como el porcentaje correspondiente a cada grupo.

Cuadro 2. Composición taxonómica de la flora vascular.

\begin{tabular}{|l|c|c|c|c|c|}
\hline Grupos & Familias & Géneros & Especies & $\begin{array}{c}\text { Unidades } \\
\text { infraespecíficas }\end{array}$ & $\begin{array}{c}\% \\
\text { de especies }\end{array}$ \\
\hline Pteridophyta & 14 & 22 & 52 & & 7 \\
\hline Gymnospermae & 2 & 4 & 14 & 2 & 2 \\
\hline Angiospermae & 12 & 56 & 119 & 1 & 17 \\
Monocotyledoneae & 80 & 225 & 531 & 13 & 74 \\
Dicotyledoneae & 108 & 307 & 716 & 16 & 100 \\
\hline Total & & & &
\end{tabular}

Los helechos y grupos afines están representados por 52 especies (7\%) del total de la flora, lo que está en función del hecho de que el área de estudio es una zona con bosques húmedos donde se presenta gran diversidad de pteridofitas, además de que un conjunto significativo perteneciente a este tipo de plantas está colonizando las áreas cubiertas por la lava.

No obstante que las gimnospermas representan sólo $2 \%$ del total de la riqueza inventariada, son muy importantes ya que dominan la fisonomía del paisaje, merced a su talla y abundancia. Dentro de las angiospermas, las monocotiledóneas conforman 17\% de 
la flora, porcentaje esperado en los bosques templados, de acuerdo con Rzedowski y Calderón de Rzedowski (1989). Las dicotiledóneas con 74\% son las más numerosas, por ser las plantas mas diversificadas y que forman siempre el mayor grupo.

En el cuadro 3 se enlistan las familias mejor representadas, tanto por el número de géneros como de especies. Se observa que en 10 familias se agrupan 127 géneros y 385 especies, que constituyen $53.4 \%$ del total registrado.

Cuadro 3. Familias con mayor número de especies en la flora.

\begin{tabular}{|l|c|c|c|}
\hline Familia & Número de géneros & Número de especies & \% de especies \\
\hline Compositae & 38 & 135 & 18.8 \\
Leguminosae & 20 & 58 & 8.1 \\
Gramineae & 28 & 57 & 7.9 \\
Labiatae & 7 & 26 & 3.6 \\
Solanaceae & 5 & 21 & 2.9 \\
Orchidaceae & 10 & 20 & 2.8 \\
Polypodiaceae & 4 & 18 & 2.5 \\
Fagaceae & 1 & 17 & 2.3 \\
Scrophulariaceae & 8 & 17 & 2.3 \\
Rosaceae & 6 & 16 & 2.2 \\
\hline Total & 127 & 385 & 53.4 \\
\hline
\end{tabular}

Rzedowski y Calderón de Rzedowski (1989) señalaron que esta composición cuantitativa de familias corresponde a una mayor afinidad hacia floras de climas templados y que el elevado número de compuestas (135) se considera como muy propio de las regiones montañosas y zonas áridas y semiáridas de México.

El cuadro 4 da cuenta de los géneros con mayor número de especies, destacando Salvia, Quercus y Eupatorium. Los encinos (Quercus) con 17 especies y los pinos (Pinus) con 11 , representan más de $50 \%$ y $70 \%$ respectivamente, del total registrado para los dos géneros en el estado de Michoacán por Madrigal (1982), así como por Bello y Labat (1987).

En el cuadro 5 se resume la importancia cuantitativa de las formas de vida, sobresaliendo las herbáceas perennes con cerca de la mitad de las especies (45.0\%), seguidas por los arbustos y subarbustos con 19.1\%, las herbáceas anuales con $15.7 \%$ y los árboles con 10.0\%; el resto se encuentra escasamente representado. Dicha distribución coincide en general con la registrada en los bosques templados de México.

Por último, se puede deducir que en el área de la comunidad indígena de Nuevo San Juan Parangaricutiro, existe una flora de clima templado, ligado a las elevaciones del 
Eje Volcánico Transversal y que sólo en la parte meridional del territorio comunal se presentan algunas especies de climas cálidos de afinidad más bien tropical.

Cuadro 4. Los géneros que se presentan con mayor número de especies.

\begin{tabular}{|l|c|l|c|}
\hline \multicolumn{1}{|c|}{ Género } & Número de especies & Género & Número de especies \\
\hline Salvia & 17 & Muhlenbergia & 11 \\
Quercus & 17 & Stevia & 11 \\
Eupatorium & 15 & Pinus & 11 \\
Senecio & 12 & Polypodium & 10 \\
Gnaphalium & 12 & Solanum & 10 \\
\hline
\end{tabular}

Cuadro 5. Importancia cuantitativa de las formas biológicas y su participación proporcional en la flora vascular de la comunidad indígena de Nuevo San Juan Parangaricutiro.

\begin{tabular}{|l|c|c|}
\hline Forma biológica & Número de especies & $\%$ \\
\hline Árboles & 73 & 10.0 \\
Arbustos y subarbustos & 140 & 19.1 \\
Herbáceas perennes & 329 & 45.0 \\
Herbáceas anuales & 115 & 15.7 \\
Trepadoras & 31 & 4.2 \\
Epífitas & 24 & 3.3 \\
Parásitas & 13 & 1.8 \\
Saprófitas & 4 & 0.5 \\
Acuáticas & 3 & 0.4 \\
\hline Total & 732 & 100.0 \\
\hline
\end{tabular}

\section{AGRADECIMIENTOS}

Nuestro reconocimiento al Dr. Jerzy Rzedowski por la revisión de la identificación de una parte de los ejemplares botánicos y por la corrección del manuscrito. Gracias asimismo a las siguientes personas: Dr. Alejandro Velázquez, Biól. Horalia Díaz-Barriga, 
a los revisores anónimos y a todas las personas que nos acompañaron al campo, entre ellas, M.C. Alejandro Torres y Biól. Magdalena Pacheco.

\section{LITERATURA CITADA}

Anónimo. 1981. Carta hidrológica de aguas superficiales. Escala 1:250 000 Hoja Colima (E 13-3). Comisión de Estudios del Territorio Nacional. México, D.F.

Anónimo. 1987. Carta topográfica. Hoja (E 13 B 39). Uruapan, Mich. Escala 1:50 000. Instituto Nacional de Estadística, Geografía e Informática. Aguascalientes, Ags.

Anónimo. 1990. Carta topográfica. Hoja (E 13 B 29). Paracho, Mich. Escala 1:50 000. Instituto Nacional de Estadística, Geografía e Informática. Aguascalientes, Ags.

Beaman, H. 1960. Vascular plants on the cinder cone of Paricutín volcano in 1958. Rhodora 61: 175186.

Bello, M. A. 1984. Estudio de muérdagos (Loranthaceae) en la región tarasca, Michoacán. Bol. Tec. Inst. Nac. Invest. For. No. 102. México, D.F. 62 pp.

Bello, M. A. 1985. Claves para la identificación de la familia Loranthaceae en la porción del Eje Neovolcánico localizado dentro del estado de Michoacán. Ciencia Forestal 54(10): 3-33.

Bello, M. A. 1993. Plantas útiles no maderables en la Sierra Purépecha, Michoacán, México. Bol. Téc. Inst. Nac. Invest. For. No. 10 México, D.F. 115 pp.

Bello, M. A. y J. N. Labat. 1987. Los encinos (Quercus) del estado de Michoacán, México. Secretaría de Agricultura y Recursos Hidráulicos. Instituto Nacional de Investigaciones Forestales y Agropecuarias y Centre d'Études Mexicaines et Centroaméricaines. Collection Études Mesoaméricaines, II. México, D.F. 98 pp.

Bello, M. A. y X. Madrigal. 1996. Estudio florístico del Campo Experimental "Barranca del Cupatitzio", Uruapan, Michoacán. Instituto Nacional de Investigaciones Forestales y Agropecuarias. Folleto Científico No. 2. México, D.F. 47 pp.

Bocco, V., A. Torres, A. Velázquez y C. Siebe. 1998. Geomorfología y recursos naturales en comunidades rurales. El caso de Nuevo San Juan Parangaricutiro, Michoacán. Geografía y Desarrollo 16: 71-84.

Delgado, P. 1992. Aspectos biológicos de conos y semillas de tres especies de Pinus, en la zona boscosa de Nuevo San Juan Parangaricutiro, Michoacán, México. Tesis de Licenciatura. Escuela de Biología. Universidad Michoacana de San Nicolás de Hidalgo. Morelia, Michoacán. 71 pp.

Eggler, W. A. 1948. Plant communities in the vicinity of the volcano El Paricutín, México, after two and a half years of eruption. Ecology 29(4): 415-436.

Eggler, W. A. 1959. Manner of invasion of volcanic deposits by plants, with further evidence from Paricutin and Jorullo. Ecol. Monogr. 29: 267-284.

Eggler, W. A. 1963. Plant life of Paricutin volcano, Mexico. Eight years after activity ceased. Amer. Midl. Nat. 69:38-68.

Espinosa, J. y L. S. Rodríguez. 1995. Listado florístico del estado de Michoacán. Sección II. Flora del Bajío y de Regiones Adyacentes. Fascículo complementario VII. Instituto de Ecología, A.C. Pátzcuaro, Mich. 242 pp.

Espinosa, J. y L. S. Rodríguez. 1996. Listado florístico del estado de Michoacán. Sección IV. Flora del Bajío y de Regiones Adyacentes. Fascículo complementario XII. Instituto de Ecología, A.C. Pátzcuaro, Mich. $271 \mathrm{pp}$.

Giménez de Azcárate, J., M. E. Escamilla y A. Velázquez. 1997. Fitosociología y sucesión en el volcán Paricutín (Michoacán, México). Caldasia 19(3): 487-505.

Hernández, F. 1959. Historia natural de Nueva España. Universidad Nacional Autónoma de México. Vol. I. México, D.F. 554 pp. 
Hernández, F. 1959. Historia natural de Nueva España. Universidad Nacional Autónoma de México. Vol. II. México, D.F. 476 pp.

Hoogstrall, H. 1941. The Río Tepalcatepec-Cerro Tancítaro transect of Michoacán, México. Urbana, Illinois. $10 \mathrm{pp}$.

Leavenworth, W.C. 1946. A preliminary study of the vegetation of de region between Cerro Tancítaro an the Río Tepalcatepec, Michoacán. México. Amer. Midl. Nat. 36: 137-206.

Madrigal, X. 1982. Claves para la identificación de las coníferas silvestres del estado de Michoacán. Instituto Nacional de Investigaciones Forestales. Boletín Divulgativo 58. México, D.F. 100 pp.

Martínez, M. A. 1997. Contribución al conocimiento de las plantas vasculares en los bosques de la comunidad indígena de Nuevo San Juan Parangaricutiro, Michoacán, México. Tesis de Licenciatura. Facultad de Biología. Universidad Michoacana de San Nicolás de Hidalgo. Morelia, Mich. $70 \mathrm{pp}$.

Moheno, C. 1985. Las historias y los hombres de San Juan. Colegio de Michoacán. Consejo Nacional de Ciencia y Tecnología. Guadalajara. 187 pp.

Rejmánek, M., R. Haagerová y J. Haager. 1982. Progress of plant succession on the Parícutin Volcano: 25 years after activity ceased. Amer. Midl. Nat. 108: 194-199.

Rodríguez, L. S. y J. Espinosa. 1995. Listado florístico del estado de Michoacán. Sección I. Flora del Bajío y de Regiones Adyacentes. Fascículo complementario VI. Instituto de Ecología A.C. Pátzcuaro, Mich. 208 pp.

Rodríguez, L. S. y J. Espinosa. 1996. Listado florístico del estado de Michoacán. Sección III. Flora del Bajío y de Regiones Adyacentes. Fascículo complementario X. Instituto de Ecología A.C. Pátzcuaro, Mich. 296 pp.

Rodríguez, L. S. y J. Espinosa. 1996. Listado florístico del estado de Michoacán. Sección V. Flora del Bajío y de Regiones Adyacentes. Fascículo complementario XV. Instituto de Ecología A.C. Pátzcuaro, Mich. 344 pp.

Rzedowski, J. 1978. Vegetación de México. Editorial Limusa. México, D.F. 432 pp.

Rzedowski, J. y G. Calderón de Rzedowski. 1989. Sinopsis numérica de la flora fanerogámica del Valle de México. Acta Bot. Mex. 8:15-30.

Rzedowski, J. y R. McVaugh. 1966. La vegetación de Nueva Galicia. Contr. Univ. Mich. Herb. 9: 1123. 


\section{APÉNDICE}

Listado florístico en el área de la comunidad indígena Nuevo San Juan Parangaricutiro, Michoacán, México.

\begin{tabular}{|c|c|c|c|}
\hline Especie & Vegetación & $\begin{array}{c}\text { Altitud } \\
\text { (en metros) }\end{array}$ & $\begin{array}{l}\text { Forma } \\
\text { de vida }\end{array}$ \\
\hline
\end{tabular}

\section{PTERIDOPHYTA}

ADIANTACEAE

Adiantum andicola Liebm.

Adiantum braunii Mett. ex Kuhn

Adiantum concinnum Humb. \& Bonpl. ex Willd.

Adiantum poiretii Wikstr.

Cheilanthes farinosa (Forssk.) Kaulf.

Cheilanthes incana (C. Presl) Mickel \& Beitel

Cheilanthes lendigera (Cav.) Sw.

Cheilanthes sinuata (Lag. ex Sw.) Domin

Pellaea ternifolia (Cav.) Link

Pteris cretica L.

Pteris orizabae M. Martens \& Galeotti

ASPLENIACEAE

* Asplenium castaneum Schltdl. \& Cham. Asplenium fragrans Sw.

Asplenium monanthes $\mathrm{L}$.

Asplenium munchii A. R. Smith

Asplenium praemorsum Sw.

ATHYRIACEAE

Cystopteris fragilis (L.) Bernh.

Woodsia mollis (Kaulf.) J. Smith

BLECHNACEAE

Woodwardia spinulosa M. Martens \& Galeotti

\section{DENNSTAEDTIACEAE}

Pteridium arachnoideum (Kaulf.) Maxon

Pteridium feei (Schaff. ex Fée) Faull

\section{DRYOPTERIDACEAE}

Dryopteris rosii C. Chr.

Dryopteris wallichiana (Spreng.) Hyl.

Plecosorus speciosissimus (A. Braun ex Kunze) Moore

\begin{tabular}{|c|c|}
\hline $\begin{array}{l}\text { BP,BOP,BPO,LIT,BMM } \\
\text { BOP }\end{array}$ & $\begin{array}{l}2500-2750 \\
3000\end{array}$ \\
\hline BMM & 1900 \\
\hline BMM,BP,BPE & $1900-2600$ \\
\hline $\mathrm{BPE}$ & 2400 \\
\hline BP & 2600 \\
\hline LIT & $2350-2400$ \\
\hline BMM & 1900 \\
\hline LIT & 2400 \\
\hline BMM & $1900-2350$ \\
\hline BMM & 2500 \\
\hline BO,BE & \\
\hline BMM & 1900 \\
\hline BPE,BMM,BOP & $2400-3100$ \\
\hline $\mathrm{BC}$ & 2500 \\
\hline LIT,BP,BPE & $2400-2600$ \\
\hline BP,BMM & $2300-2400$ \\
\hline BPE,BPO & $2400-2900$ \\
\hline BPO & 2350 \\
\hline BMM,BPE & $1900-2500$ \\
\hline BPE & 2500 \\
\hline LIT & 2400 \\
\hline BMM,BOP & $2500-2950$ \\
\hline BOP & 3200 \\
\hline
\end{tabular}


Apéndice. Continuación.

Especie
EQUISETACEAE
Equisetum hyemale var. affine (Engelm.)

A. A. Eaton

BMM

1900

HP

LOMARIOPSDIACEAE

Elaphoglossum petiolatum (Sw.) Urb.

Elaphoglossum piloselloides (C. Presl) Moore

Vegetación

Altitud

Forma

(en metros) de vida

Elaphoglossum tambillense (Hook.) Moore, Mickel \& Beitel

$\begin{array}{lll}\text { BMM,BPE } & 2300-2450 & \text { HP } \\ \text { BMM } & 1900 & \text { HP } \\ \text { BMM } & 2400 & \text { HP } \\ \text { BMM } & & \\ \text { BMM } & 2600 & \text { EP } \\ & 2400 & \text { HP }\end{array}$

Botrychium virginianum

(L.) Sw.

BMM

LIT

LIT

2400

HP

Galeotti) Mickel \& Beitel

* Phlebodium aureum (L.) Sm.

Pleopeltis angusta Humb. \& Bonpl. ex Willd.

Pleopeltis interjecta (Weath.) Mickel \& Beitel

BMM,LIT

$2400-2550$

HP

BPE

2500

EP

* Pleopeltis macrocarpa (Bory) Kaulf. ex Willd.

BP

Pleopeltis mexicana (Fée) Mickel \& Beitel

Pleopeltis polylepis (Roem. ex Kunze) Moore

BPE

2500

EP

Polypodium fraternum Schltdl. \& Cham.

Polypodium hartwegianum Hook.

Polypodium madrense J. Smith

Polypodium martensii Mett.

Polypodium platylepis Mett. ex Kuhn

Polypodium plebeium Schltdl. \& Cham.

* Polypodium plectolepis Hook.

Polypodium polypodioides (L.) Watt

Polypodium subptetiolatum Hook.

Polypodium thyssanolepis A. Braun ex Klotzsch

Polystichum rechichlaena Fée

BE,BPE

$2400-2800$

EP

$B P O$

2400

$\mathrm{BP}$

$\mathrm{BMM}, \mathrm{BOP}, \mathrm{BE}$

2600

BPE

2500-2800

2600

BMM

2400-2550

BMM

BP,BE,BPE,BMM,MS

BMM

BPE

1900

HP

LIT

BMM

1900

2400

EP

EP

EP

EP

EP

EP

EP

$\mathrm{HP}$

EP

HP

2400

2450

HP

$\mathrm{HP}$

SELAGINELLACEAE

Selaginella delicatissima Linden ex A.

Braun

BMM

1900

HP 
Acta Botanica Mexicana (2000), 52: 5-41

Apéndice. Continuación.

\begin{tabular}{lccc}
\hline Especie & Vegetación & $\begin{array}{c}\text { Altitud } \\
\text { (en metros) }\end{array}$ & $\begin{array}{c}\text { Forma } \\
\text { de vida }\end{array}$ \\
\hline $\begin{array}{l}\text { Selaginella pallescens (C. Presl) Spring } \\
\text { THELYPTERIDACEAE } \\
\begin{array}{l}\text { Thelypteris pilosa (M. Martens \& Galeotti) } \\
\quad \text { Crawford }\end{array}\end{array}$ & BMM & 1900 & HP \\
$\begin{array}{l}\text { VITTARIACEAE } \\
\text { Vitttaria flavicosta Mickel \& Beitel }\end{array}$ & BMM & 1900 & HP \\
\end{tabular}

\section{GYMNOSPERMAE}

CUPRESSACEAE

Cupressus lusitanica Mill.

Juniperus monticola Martínez

BPE,REF

PAZ

2400-2600

2800

AR

$A B$

PINACEAE

Abies religiosa (HBK.) Schltdl. \& Cham. var. religiosa

Abies religiosa var. emarginata Loock \& Martínez

Pinus devoniana Lindl.

Pinus douglasiana Martínez

Pinus hartwegii Lindl.

* Pinus lawsonii Roezl

Pinus leiophylla Schltdl. \& Cham.

Pinus maximinoi $\mathrm{H}$. E. Moore

Pinus montezumae Lamb.

* Pinus oocarpa Schiede ex Schltdl.

* Pinus pringlei Shaw

Pinus pseudostrobus Lindl.

Pinus pseudostrobus f. protuberans Martínez

Pinus teocote Schltdl. \& Cham.

\section{ANGIOSPERMAE}

\section{ACANTHACEAE}

Dyschoriste angustifolia (Hemsl.) Kuntze

AMARANTHACEAE

Iresine diffusa Humb. \& Bonpl. ex Willd.

AMARYLLIDACEAE

Agave inaequidens K. Koch

Bomarea hirtella (HBK.) Herb.

Furcraea bedinghausii K. Koch
BP

2150

HP

BMM,BPE,BOP

$2400-2550$

HP

$\begin{array}{lll}\text { BPE,BO } & 2400-3200 & \text { AR } \\ \text { BOP,BMM } & 2500-3200 & \text { AR } \\ \text { BP } & 2600-2800 & \text { AR } \\ \text { BP,BPE,BO } & 2350-2950 & \text { AR } \\ \text { BC } & 2800 & \text { AR } \\ \text { BP } & 2750 & \text { AR } \\ \text { BP,BPE,BPO } & 2300-2800 & \text { AR } \\ \text { BP,BPE,BO,BPO,PAZ, } & 2400-2950 & \text { AR } \\ \text { LIT,REF } & & \text { AR } \\ & & \text { AR } \\ \text { BMM,BP,BPE,BPO,REF } & 2150-3100 & \text { AR } \\ & & \\ \text { BC } & 2600 & \text { AR } \\ & & \text { AR }\end{array}$

BPE

2550

RUD,BMM

2700
HA

TR

AB 
Apéndice. Continuación.

\section{Especie}

\section{Vegetación}

Begonia sp.

\section{BERBERIDACEAE}

Berberis moranensis Hebenstr. \& Ludw. ex Schult.

\section{BETULACEAE}

Alnus acuminata ssp. arguta (Schltdl.) Furlow

Alnus acuminata ssp. glabrata (Fernald) Furlow

Alnus jorullensis HBK. ssp. jorullensis

Alnus jorullensis ssp. lutea Furlow

Carpinus caroliniana Walter

BORAGINACEAE

Hackelia mexicana (Schltdl. \& Cham.)

I. M. Johnst.

* Lasiarrhenum oblongifolium Greenm.

* Lithospermum strigosum (HBK.) I. M. Johnst.

Macromeria longiflora (Sessé \& Moc.)

D. Don

Tournefortia hirsutissima L.

Tournefortia petiolaris DC.
BPE

BMM

BMM

2000

2000

BMM

BMM

$\mathrm{BP}$

BP,PAZ,BPE

$B P, P A Z, B P E$

BMM

BMM

BMM

RUD,BMM,BE,BOP

2600-3000

2450-2550

2150

2300-2700

2300-2700

2400

2550

1900

HA

Altitud

Forma

de vida

TR

AB

AR

AR

$A R$

$A B$

\section{$A B$}

$A B$

TR

HP

$\begin{array}{lll}\text { BMM } & 2300 & \text { AR } \\ \text { BMM } & 1900 & \text { AR } \\ \text { BPE,BO } & 2400-3200 & \text { AR } \\ \text { BPO,BP,BPE } & 2400-2800 & \text { AR } \\ \text { BMM } & 1900 & \text { AR }\end{array}$

BO

3200

AB

BE

$\mathrm{HP}$

BP

HP

BO

2900

$A B$

BMM

2300

$A B$

BMM

1900

$A B$ 
Acta Botanica Mexicana (2000), 52: 5-41

Apéndice. Continuación.

Especie
Vegetación

Altitud (en metros)

Forma de vida

BROMELIACEAE

Tillandsia macdougallii L. B. Sm.

Tillandsia prodigiosa (Lem.) Baker

* Tillandsia usneoides (L.) L.

Tillandsia violacea Baker

CACTACEAE

Heliocereus sp.

CAMPANULACEAE

Diastatea micrantha (HBK.) McVaugh

Lobelia fenestralis Cav.

Lobelia laxiflora HBK. var. laxiflora

* Lobelia laxiflora var. angustifolia A. DC. Lobelia nana HBK.

Lobelia plebeia E. Wimm.

CAPRIFOLIACEAE

Sambucus mexicana C. Presl ex A. DC.

Symphoricarpos microphyllus HBK.

Viburnum acutifolium ssp. microphyllum

(Oerst.) Donoghue

Viburnum elatum Benth.

Viburnum lautum C. V. Morton

CARYOPHYLLACEAE

Arenaria lanuginosa (Michx.) Rohrb.

Arenaria reptans Hemsl.

Cerastium molle Bartl.

Cerastium nutans Raf.

Drymaria glandulosa Bartl.

Drymaria malachioides Briq.

Drymaria villosa Cham. \& Schltdl.

Spergularia mexicana Hemsl.

Stellaria cuspidata Willd. ex Schltdl.

Stellaria media (L.) Cirillo

\section{CHENOPODIACEAE}

Chenopodium sp.

CHLORANTHACEAE

Hedyosmum mexicanum Cordem.

CISTACEAE

Helianthemum glomeratum (Lag.) Lag. ex Dunal Helianthemum sp.

\begin{tabular}{|c|c|c|}
\hline \multirow{2}{*}{$\begin{array}{l}B C \\
B C\end{array}$} & 2700 & $\mathrm{HP}$ \\
\hline & 2500 & $\begin{array}{l}\text { EP } \\
\text { EP }\end{array}$ \\
\hline BPO & 2500 & EP \\
\hline BMM & 1900 & EP \\
\hline BMM & 1900 & $\mathrm{HA}$ \\
\hline PAS & 2300 & $\mathrm{HA}$ \\
\hline BP,BPE & $2400-2650$ & HP \\
\hline BP & & HP \\
\hline BC & 3000 & HP \\
\hline BC & 2650 & $\mathrm{HA}$ \\
\hline BPO & $2700-2800$ & AR \\
\hline BO & 2800 & $A B$ \\
\hline BMM,BP & $2300-2600$ & AR \\
\hline BMM & 2450 & AR \\
\hline BC & 2600 & AR \\
\hline BPO,BMM,BP & $2300-2800$ & $\mathrm{HP}$ \\
\hline BPO,BP,BO & $2300-3100$ & HP \\
\hline PAZ & 2800 & $\mathrm{HP}$ \\
\hline BPE,BMM,BO & $2300-3100$ & $\mathrm{HA}$ \\
\hline BMM & 1900 & $\mathrm{HA}$ \\
\hline BPE & $2500-2900$ & $\mathrm{HA}$ \\
\hline BPE & 2600 & $\mathrm{HA}$ \\
\hline ARV & 2800 & HP \\
\hline BOP,BP,BMM,ARV,BPE & $2300-3200$ & HP \\
\hline BP & 2650 & $\mathrm{HA}$ \\
\hline MS & 2400 & HP \\
\hline BMM & 1900 & $A B$ \\
\hline MS & 2500 & $\begin{array}{l}\text { HP } \\
\text { HP }\end{array}$ \\
\hline
\end{tabular}


Apéndice. Continuación.

\begin{tabular}{|c|c|c|c|}
\hline Especie & Vegetación & $\begin{array}{c}\text { Altitud } \\
\text { (en metros) }\end{array}$ & $\begin{array}{l}\text { Forma } \\
\text { de vida }\end{array}$ \\
\hline
\end{tabular}

CLETHRACEAE

Clethra hartwegii Britton

* Clethra mexicana A. DC.

Acourtia michoacana (B. L. Rob.) Reveal

COMPOSITAE \& R. M. King

* Acourtia turbinata (La Llave \& Lex.) Reveal \& R. M. King

Ageratum corymbosum Zuccagni

Archibaccharis asperifolia (Benth.) S. F. Blake

Archibaccharis hieraciifolia Heering

Archibaccharis schiedeana (Benth.) J. D. Jackson

Archibaccharis serratifolia (HBK.) S. F. Blake

Astranthium orthopodum (B. L. Rob. \& Fernald) Larsen

Baccharis conferta HBK.

Baccharis heterophylla HBK.

Baccharis multiflora HBK.

Baccharis pteronioides DC.

Baccharis serraefolia DC.

Bidens aequisquama (Fernald) Sherff

Bidens aurea (Aiton) Sherff

Bidens ferulifolia (Jacq.) DC.

* Bidens odorata Cav.

Bidens ostruthioides (DC.) Sch. Bip.

* Bidens serrulata (Poir.) Desf.

* Bidens triplinervia HBK.

BPE
BO,BP,BPE,BMM,
BP,BE,BMM,MS
BP,BPE
BP,BPE,ARV
PAS
BMM
BE
BO

PAS,BMM

BMM

2650

AR

AR

$\mathrm{HP}$

2450-2500 HP

2050-2800 HA

$2300 \quad$ HP

1900-2500 HP

-

HA

1900-2300 HP

2400

HA

BPO

3000

HP

BPE

BPE

2450

HP

BP

2600

2650

$A B$

BC

1900-2500

$A B$

BMM

1900

$A B$

BP

BO,BP

2600

2750-2900

2400-2900

2800

2500

2400-2650

2300-2800

2500

2200

HA

$\mathrm{BO}$

BPE,BMM,MS

BPE,BMM,BPO

BMM,BPE

BPE

$B O, B P, B E$

BP,BMM,BO

$\mathrm{BP}, \mathrm{BE}$

$\mathrm{BO}, \mathrm{BP}, \mathrm{BE}, \mathrm{BPE}$

1900-2600

$A B$

$A B$

$A B$

$A B$

$A B$

$\mathrm{HA}$

HP

HA

HA

HP

HA

HP 
Acta Botanica Mexicana (2000), 52: 5-41

Apéndice. Continuación.

\begin{tabular}{|c|c|c|c|}
\hline Especie & Vegetación & $\begin{array}{c}\text { Altitud } \\
\text { (en metros) }\end{array}$ & $\begin{array}{l}\text { Forma } \\
\text { de vida }\end{array}$ \\
\hline
\end{tabular}

Brickellia pedunculosa (DC.) Harc. \& Beaman

* Brickellia pendula (Schrad.) A. Gray Brickellia squarrosa (Cav.) B. L. Rob. Calea integrifolia (DC.) Hemsl.

* Calea scabra (Lag.) B. L. Rob. Calea urticifolia (Mill.) DC.

* Cirsium anartiolepis Petr. Cirsium ehrenbergii Sch. Bip.

Cirsium nivale (HBK.) Sch. Bip.

Cirsium velatum (S. Watson) Petr.

Cirsium tolucanum (B. L. Rob. \& Seaton) Petr.

Conyza canadensis (L.) Cronquist

Conyza coronopifolia HBK.

* Conyza microcephala Hemsl.

Conyza schiedeana (Less.) Cronquist

* Conyza sophiifolia HBK.

Cosmos bipinnatus Cav.

* Cosmos carvifolius Benth.

Dahlia coccinea Cav.

Dahlia rudis P. D. Sorensen

Dahlia scapigera (A. Dietr.) Knowles \& Westc.

* Erigeron delphinifolius Willd.

Erigeron galeottii (A. Gray ex Hemsl.) Greene

Erigeron polycephalus (Larsen) G. L. Nesom

BPE
BP
BPE
BP
BP
BO
BO
BPO,BPE
BC
BO
BP,PAZ,BOP
BPE,ARV
BP
BP
PAZ
BP
MS
BP
BMM
BP
BC
BP,BMM
BPE
BC
BO
ARV
BMM
BP
ARV
BMM
BMM
BPO,BP,BMM
BO,BP,BE,BPE,BMM
BPE
BP
BC
BOP,BO,PAZ
BO
BC
BMM,BPO

2350

HP

2400

2150

2800

$A B$

$A B$

$A B$

$\mathrm{HP}$

$\mathrm{AB}$

2650-2750

2800

2850

$\mathrm{HP}$

$\mathrm{HP}$

$\mathrm{HP}$

HP

2800-3200 HP

2150-2450 HA

3000

HA

2500

HP

HP

2500

$\mathrm{HA}$

$\mathrm{HA}$

AB

$2300 \quad \mathrm{HP}$

2000 HP

* Erigeron pubescens HBK.

Erigeron velutipes Hook. \& Arn.

Eupatorium areolare DC.

Eupatorium arsenei B. L. Rob.

Eupatorium aschenbornianum S. Schauer

* Eupatorium brevipes DC.

Eupatorium dolichobasis McVaugh

Eupatorium glabratum HBK.

* Eupatorium isolepis B. L. Rob.

Eupatorium mairetianum DC.

Eupatorium malacolepis B. L. Rob.

Eupatorium oresbium B. L. Rob.

Eupatorium pazcuarense HBK.

Eupatorium petiolare Moc. ex DC.

Eupatorium pycnocephalum Less.

Eupatorium rivale Greenm.

$\mathrm{BMM}, \mathrm{BPO}$

2800

HP

HA

$2400 \quad$ HP

$2500 \quad \mathrm{HP}$

$2150-1$

1900-2450 AB

$2150 \quad \mathrm{HP}$

$2150 \quad \mathrm{HP}$

$2450 \quad \mathrm{HP}$

2350-3000 AB

$2400-H P$

$2150-A B$

$2350 \quad A B$

2700-3200 HP

$2800 \quad A B$

$2800 \quad \mathrm{HP}$

2450-2800 AB 
Apéndice. Continuación.

\begin{tabular}{|c|c|c|c|}
\hline Especie & Vegetación & $\begin{array}{c}\text { Altitud } \\
\text { (en metros) }\end{array}$ & $\begin{array}{l}\text { Forma } \\
\text { de vida }\end{array}$ \\
\hline
\end{tabular}

* Eupatorium rubricaule HBK. Galinsoga quadriradiata Ruiz \& Pav. Gnaphalium americanum Mill. Gnaphalium attenuatum DC. Gnaphalium bourgovii A. Gray Gnaphalium canescens DC. Gnaphalium inornatum DC. Gnaphalium liebmannii Sch. Bip. ex Klatt

* Gnaphalium oxyphyllum DC. Gnaphalium roseum HBK. Gnaphalium salicifolium (Bertol.) Sch. Bip. Gnaphalium semiamplexicaule DC. Gnaphalium stramineum HBK.

* Gnaphalium viscosum HBK. Heterotheca inuloides Cass. var inuloides Heterotheca inuloides var. rosei B. Wagenkn.

Hieracium abscissum Less.

Hieracium schultzii Fr.

* Jaegeria bellidiflora (DC.) A. M. Torres \& Beaman Jaegeria hirta (Lag.) Less. Jaegeria macrocephala Less. Lagascea helianthifolia HBK. Melampodium montanum Benth.

* Melampodium perfoliatum (Cav.) HBK.

* Montanoa frutescens Mairet ex DC.

Perymenium alticola McVaugh

* Perymenium buphthalmoides DC. var. buphthalmoides

Perymenium buphthalmoides var. flexuosum (Greenm.) McVaugh

Pinaropappus roseus (Less.) Less.

Piqueria pilosa HBK.

Piqueria triflora Hemsl.

Piqueria trinervia Cav.

Podachaenium eminens (Lag.) Sch. Bip.

Rumfordia floribunda DC.

Sabazia humilis (HBK.) Cass.

Sabazia liebmannii Klatt

Senecio albonervius Greenm.

Senecio angulifolius DC.

Senecio barba-johannis DC.

Senecio callosus Sch. Bip.

Senecio mexicanus McVaugh

\begin{tabular}{|c|c|c|}
\hline $\mathrm{BE}$ & & $A B$ \\
\hline ARV & 2150 & $\mathrm{HA}$ \\
\hline ARV,MS & $2150-2800$ & $\mathrm{HA}$ \\
\hline $\mathrm{BO}, \mathrm{BPO}$ & 2800 & HP \\
\hline $\mathrm{BO}$ & 2850 & $\mathrm{HA}$ \\
\hline BP & 2400 & HP \\
\hline BMM & 1900 & HP \\
\hline RUD & 2300 & HP \\
\hline BMM & & $\mathrm{HA}$ \\
\hline BO & 2850 & HP \\
\hline BP & 2600 & HP \\
\hline MS,BO,PAS,BPE & $2450-3200$ & HP \\
\hline BPE & 2500 & $\mathrm{HA}$ \\
\hline $\mathrm{BO}, \mathrm{BP}, \mathrm{MS}$ & & $\mathrm{HA}$ \\
\hline BPO,ARV,MS & $2150-2800$ & HP \\
\hline BPE & 2300 & HP \\
\hline BPE & 2650 & HP \\
\hline BO & 3200 & $\mathrm{HA}$ \\
\hline BP & & HP \\
\hline BPE,BP,ARV & $2150-2600$ & $\mathrm{HA}$ \\
\hline BMM & 1900 & HP \\
\hline BMM,BPE & $1900-2550$ & $A B$ \\
\hline BP & 2150 & HP \\
\hline BP,BE & & $\mathrm{HA}$ \\
\hline $\mathrm{BO}, \mathrm{BPE}$ & & $A B$ \\
\hline $\mathrm{BC}$ & 3000 & $A B$ \\
\hline BO,BP & & $\mathrm{HP}$ \\
\hline BC & 2550 & HP \\
\hline $\mathrm{BC}$ & 2800 & $\mathrm{HA}$ \\
\hline $\mathrm{BMM}, \mathrm{BO}$ & $2400-2800$ & $\mathrm{HA}$ \\
\hline BP,BMM & $1900-2600$ & $\mathrm{HA}$ \\
\hline BO,PAZ & 3200 & HP \\
\hline LIT & 2300 & HP \\
\hline BMM & $1900-2300$ & $A B$ \\
\hline BC & 2350 & $\mathrm{HA}$ \\
\hline BO & 2800 & HP \\
\hline BPE,BE,MS & $2600-2850$ & $A B$ \\
\hline BPO,BPE,BO,BP,MS & $2450-3200$ & $A B$ \\
\hline BPE,BMM,BOP & $2450-3000$ & $A B$ \\
\hline BPO & $3100-3200$ & HP \\
\hline BPE,BMM & $2300-2600$ & $A B$ \\
\hline
\end{tabular}


Acta Botanica Mexicana (2000), 52: 5-41

Apéndice. Continuación.

\begin{tabular}{|c|c|c|c|}
\hline Especie & Vegetación & $\begin{array}{c}\text { Altitud } \\
\text { (en metros) }\end{array}$ & $\begin{array}{l}\text { Forma } \\
\text { de vida }\end{array}$ \\
\hline Senecio peltiferus Hemsl. & BPE & 2600 & $\mathrm{HP}$ \\
\hline * Senecio roldana DC. & $\mathrm{BO}, \mathrm{BP}$ & & $\mathrm{HP}$ \\
\hline Senecio salignus DC. & MS & 2600 & $A B$ \\
\hline Senecio sanguisorbae DC. & $\mathrm{BO}, \mathrm{MS}$ & 3200 & HP \\
\hline Senecio sinuatus HBK. & BPE & 2600 & $\mathrm{HP}$ \\
\hline Senecio stoechadiformis DC. & BMM,BP,MS & $2600-2700$ & $\mathrm{HP}$ \\
\hline Senecio toluccanus DC. & BPE & 2450 & $\mathrm{HP}$ \\
\hline Sigesbeckia agrestis Poepp. \& Endl. & BMM & 1900 & $\mathrm{HP}$ \\
\hline Sigesbeckia jorullensis HBK. & $\mathrm{BO}$ & $3100-3200$ & $\mathrm{HP}$ \\
\hline Sonchus oleraceus L. & ARV,BO & $2150-3000$ & $\mathrm{HP}$ \\
\hline Stevia jorullensis HBK. & $\mathrm{BO}$ & 2850 & $\mathrm{HP}$ \\
\hline Stevia lucida Lag. & $\mathrm{BC}$ & 2700 & $A B$ \\
\hline Stevia micradenia B. L. Rob. & BMM & 2400 & $\mathrm{HP}$ \\
\hline Stevia monardifolia HBK. & BMM,BPE,BP,BOP & $2400-3200$ & $\mathrm{HP}$ \\
\hline * Stevia myricoides McVaugh & $\mathrm{BP}$ & & $A B$ \\
\hline Stevia nelsonii B. L. Rob. & $\mathrm{BPO}, \mathrm{BOP}$ & $2700-3100$ & $A B$ \\
\hline * Stevia nepetifolia HBK. & $\mathrm{BPE}, \mathrm{BE}$ & & $\mathrm{HP}$ \\
\hline Stevia aff. origanoides HBK. & $\mathrm{BPE}$ & 2050 & $\mathrm{HP}$ \\
\hline Stevia ovata Willd. & BPE,BP,PAS & $2250-2600$ & $\mathrm{HP}$ \\
\hline Stevia salicifolia Cav. & BPE & 2500 & $A B$ \\
\hline Stevia subpubescens Lag. & BPE & 2550 & $A B$ \\
\hline Tagetes filifolia Lag. & ARV & 2950 & $\mathrm{HA}$ \\
\hline Tagetes foetidissima DC. & BPE,PAS,MS & 2500 & $\mathrm{HA}$ \\
\hline Tagetes remotiflora Kunze & BMM & 1900 & $\mathrm{HA}$ \\
\hline Tagetes subulata Cerv. & BMM & 2400 & $\mathrm{HA}$ \\
\hline Tagetes triradiata Greenm. & $\mathrm{BP}$ & 2600 & $\mathrm{HA}$ \\
\hline Taraxacum officinale Weber & $\mathrm{BC}$ & 2650 & $\mathrm{HA}$ \\
\hline * Tithonia tubaeformis (Jacq.) Cass. & BMM & & $\mathrm{HA}$ \\
\hline Trigonospermum melampodioides DC. & BMM & 1900 & $\mathrm{HP}$ \\
\hline Verbesina discoidea (Brandegee) Rzed. & BPO & 3100 & $A B$ \\
\hline Verbesina fastigiata B. L. Rob. \& Greenm. & BPE & 2650 & $A B$ \\
\hline Verbesina greenmanii Urb. & BMM & 1900 & $A B$ \\
\hline Verbesina klattii B. L. Rob. \& Greenm. & BMM & $2400-2550$ & $A B$ \\
\hline Verbesina oncophora B. L. Rob. \& Seaton & BMM & $1900-2400$ & $A B$ \\
\hline * Verbesina tetraptera (Ortega) A. Gray & $\mathrm{BE}$ & & $\mathrm{HP}$ \\
\hline Viguiera hemsleyana S. F. Blake & $\mathrm{BE}$ & 2600 & $\mathrm{HP}$ \\
\hline \multicolumn{4}{|l|}{ CONVOLVULACEAE } \\
\hline \multicolumn{4}{|l|}{ Cuscuta corymbosa var. grandiflora } \\
\hline Engelm. & RUD & 2700 & PA \\
\hline Cuscuta rugosiceps Yunck. & $\mathrm{BPO}$ & 2700 & PA \\
\hline Ipomoea funis Schltdl. \& Cham. & BMM & $1900-2000$ & TR \\
\hline Ipomoea purpurea (L.) Roth & ARV & 2150 & $\mathrm{HA}$ \\
\hline
\end{tabular}


Apéndice. Continuación.

\begin{tabular}{|c|c|c|c|}
\hline Especie & Vegetación & $\begin{array}{c}\text { Altitud } \\
\text { (en metros) }\end{array}$ & $\begin{array}{l}\text { Forma } \\
\text { de vida }\end{array}$ \\
\hline
\end{tabular}

CORIARIACEAE

Coriaria ruscifolia ssp. microphylla (Poir.)

L. E. Skog

BO,BP,BPE,BMM,MS $\quad 2000-2650 \quad$ AB

CORNACEAE

Cornus disciflora Sessé \& Moc. ex DC. BMM $\quad 2400-2550 \quad$ AB

CRASSULACEAE

Echeveria fulgens Lem.

Echeveria mucronata (Baker) Schltdl.

Sedum bourgaei Hemsl.

Villadia batesii (Hemsl.) Baehni \& J. F. Macbr.

$\mathrm{BC}$

$2700 \quad \mathrm{HP}$

BMM

1900-2400 HP

BPO

2800

HP

CRUCIFERAE

Brassica campestris L.

* Brassica nigra (L.) W. D. J. Koch

ARV

BP

Draba jorullensis HBK.

$\mathrm{BC}$

2800

HA

Halimolobos berlandieri (E. Fourn.) O. E. Schulz

$\begin{array}{lll}\text { PAZ } & 2800 & \text { HA }\end{array}$

* Lepidium lasiocarpum Nutt. ex Torr. \& A. Gray

Lepidium virginicum $\mathrm{L}$.

Raphanus raphanistrum $\mathrm{L}$.

Romanschulzia arabiformis (DC.) Rollins

Rorippa sp.

BO

ARV,BPE

RUD

$B O, P A Z$

SAC

2800

HA

HP

CUCURBITACEAE

* Cyclanthera dissecta (Torr. \& A. Gray) Arn. Cyclanthera langaei Cogn.

Sicyos deppei G. Don

Sicyos parviflorus Willd.

BO

BMM

ARV

BMM

$\mathrm{HA}$

CYPERACEAE

Carex peucophila Holm

Carex aff. tuberculata Liebm.

$\mathrm{BC}$

2150-2550 HA

2600 HP

$3200 \quad \mathrm{HP}$

* Cyperus hermaphroditus (Jacq.) Standl.

$\mathrm{BC}$

BP

Cyperus manimae HBK.

PAZ

2900

AC

* Cyperus sanguineoater Boeck.

BP

Cyperus seslerioides HBK.

* Cyperus sesquiflorus (Torr.) Mattf. \& Kük. Cyperus spectabilis Link

BPE,REF

BP

BPE

* Cyperus tenuifolius (Steud.) Dandy

Cyperus sp.

BP

BP

$\begin{array}{ll} & \text { TR } \\ 1900-2500 & \text { TR } \\ 2150 & \text { TR } \\ 1900 & \text { TR }\end{array}$

DILLENIACEAE

Saurauia serrata DC.

BMM

$\begin{array}{ll}2400 & \text { HP } \\ 2700 & \text { HP } \\ & \text { HP } \\ 2650 & \text { HP } \\ & \text { HP } \\ 2400-2600 & \text { HP } \\ & \text { HP } \\ 2500 & \text { HP } \\ & \text { HA } \\ 2400 & \text { HP } \\ & \\ & \\ 2000 & \text { AR }\end{array}$


Acta Botanica Mexicana (2000), 52: 5-41

Apéndice. Continuación.

\begin{tabular}{|c|c|c|c|}
\hline Especie & Vegetación & $\begin{array}{c}\text { Altitud } \\
\text { (en metros) }\end{array}$ & $\begin{array}{l}\text { Forma } \\
\text { de vida }\end{array}$ \\
\hline
\end{tabular}

ERICACEAE

Arbutus glandulosa M. Martens \& Galeotti

Arbutus occidentalis var. villosa McVaugh \& Rosatti

BPE

PAZ

BPE

BPE

Arbutus xalapensis HBK.

Arctostaphylos discolor (Hook.) DC.

Arctostaphylos pungens HBK.

Arctostaphylos rupestris B. L. Rob. \& Seaton

Gaultheria cordata ssp. lancifolia (Small)

Corcoran \& Breckon

Pernettya ciliata (Schltdl. \& Cham.) Small

Vaccinium geminiflorum HBK.

\section{EUPHORBIACEAE}

Euphorbia graminea Jacq.

* Euphorbia ocymoidea L.

Euphorbia prostrata Aiton

Euphorbia stictospora Engelm.

FAGACEAE

Quercus candicans Née

Quercus castanea Née

Quercus conspersa Benth.

Quercus crassifolia Humb. \& Bonpl.

Quercus crassipes Humb. \& Bonpl.

* Quercus deserticola Trel.

Quercus dysophylla Benth.

Quercus gentryi C. H. Müll.

Quercus glaucescens Humb. \& Bonpl.

Quercus laurina Humb. \& Bonpl.

Quercus magnoliaefolia Née

Quercus martinezii C. H. Müll.

* Quercus obtusata Humb. \& Bonpl.

Quercus peduncularis Née

Quercus planipocula Trel.

Quercus rugosa Née

Quercus splendens Née

FLACOURTIACEAE

Xylosma flexuosum (HBK.) Hemsl.

Xylosma aff. velutinum Triana \& Karst.

GARRYACEAE

Garrya laurifolia Hartw. ex Benth.
BPE,BP,MS

$\mathrm{BO}$

BPE

MS,LIT

BO,BPE

BMM

BMM

BP

BP

LIT

BMM,BPE

BMM

BE,BPO

BPE,BP

BPE

BE

BPE

BMM

BP

BPO,BPE,BE

BPE

BPE

BP,BE,BPE

BMM

BPE

BMM,BPE

BOP

BPO,BPE

BMM

BPE
2400

AR

2800

2400

2550

2450-2650

2850

AB

AR

$A R$

AB

$A B$

$2400-2500$

$A B$

2450-2500

2900-2950

2600

AB

$\mathrm{HP}$

$A B$

1900-2400

HA

$\mathrm{HA}$

2400

2400

HP

$\mathrm{HA}$

1900-2400

1900

2750

2500-2650

2400-2600

2450

2300

2150

2300-2750

2500

2550

2300

2300-2550

2900

AR

AR

AR

AR

AR

AR

AR

AR

AR

AR

AR

AR

AR

AR

AR

AR

AR

2500-2600

2400

AR

$A B$

2600

AR 
Apéndice. Continuación.

\begin{tabular}{llcc}
\hline Especie & Vegetación & $\begin{array}{c}\text { Altitud } \\
\text { (en metros) }\end{array}$ & $\begin{array}{c}\text { Forma } \\
\text { de vida }\end{array}$ \\
\hline $\begin{array}{l}\text { GENTIANACEAE } \\
\text { Centaurium quitense (HBK.) B. L. Rob. }\end{array}$ & LIT & 2400 & HA \\
$\begin{array}{l}\text { Gentianella amarella ssp. hartwegii } \\
\text { (Benth.) J. M. Gillett }\end{array}$ & BMM & 2400 & HA \\
Halenia brevicornis (HBK.) G. Don & BPE & 2650 & HA
\end{tabular}

\section{GERANIACEAE}

* Erodium cicutarium (L.) L'Hér.

* Erodium moschatum (L.) L'Hér.

* Geranium aristisepalum H. E. Moore

* Geranium cruceroense R. Knuth

Geranium latum Small

Geranium lilacinum R. Knuth

Geranium seemannii Peyr.

\section{GESNERIACEAE}

Kohleria elegans (Decne.) Loes.

BO,BP

HA

$\mathrm{BP}, \mathrm{BE}$

$\mathrm{BO}, \mathrm{BP}$

$\mathrm{BP}$

BPO,BPE

BPE,BOP

$A R V, B P E, B O P$

$\begin{array}{ll} & \text { HA } \\ & \text { HA } \\ & \text { HP } \\ & \text { HP } \\ 2700 & \text { HP } \\ 2450-3200 & \text { HP } \\ 2150-3100 & \text { HP }\end{array}$

BMM

1900

$A B$

\section{GRAMINEAE}

Aegopogon cenchroides Humb. \& Bonpl. ex Willd.

Aegopogon tenellus (DC.) Trin.

* Agrostis hyemalis (Walter) B. S. P. Agrostis aff. perennans (Walter) Tuck.

* Andropogon barbinodis Lag.

* Aristida divaricata Humb. \& Bonpl. ex Willd.

* Bouteloua curtipendula (Michx.) Torr. Brachypodium mexicanum (Roem. \& Schult.) Link

* Bromus anomalus Rupr. ex Fourn. Bromus carinatus Hook. \& Arn. Bromus exaltatus Bernh.

Bromus dolichocarpus Wagnon

* Bromus inermis Leyss. Calamagrostis valida Sohns Chloris sp.

Chusquea aff. circinata Soderstrom \& Calderón

BPE,BP,LIT,PAS,MS

BPE

BP

ARV

BP

MS

BP

BPE,BOP,RUD,ARV

$B E$

BOP

$\mathrm{BC}$

BMM,BPE,ARV

BMM

BPE

ARV

BC

ARV,MS

BP,MS

* Digitaria leucites (Trin.) Henrard

Eragrostis mexicana (Hornem.) Link ssp. mexicana

Eragrostis pectinacea (Michx.) Nees

Festuca amplissima Rupr.

Festuca breviglumis Swallen

$\begin{array}{lll}\text { ARV } & 2150 & \text { HA } \\ \text { LIT } & 2150 & \text { HA } \\ \text { BO,PAZ } & 3150 & \text { HP } \\ \text { BP,BMM,BPE } & 2400-2500 & \text { HP }\end{array}$

$\begin{array}{ll}2400-2600 & \text { HA } \\ 2500 & \text { HA } \\ 2800 & \text { HP } \\ & \text { HP } \\ & \text { HP } \\ & \text { HP } \\ & \text { HP } \\ & \\ 2150-3000 & \text { HP } \\ & \text { HP } \\ 3200 & \text { HP } \\ 2850 & \text { HA } \\ 2300-2600 & \text { HP } \\ & \text { HP } \\ 2550 & \text { HP } \\ 2150 & \text { HA } \\ & \\ 2650 & \text { AB } \\ 2150-2400 & \text { HP } \\ & \text { HP } \\ 2150 & \text { HA } \\ 2150 & \text { HA } \\ 3150 & \text { HP } \\ 2400-2500 & \text { HP }\end{array}$


Acta Botanica Mexicana (2000), 52: 5-41

Apéndice. Continuación.

\begin{tabular}{|c|c|c|c|}
\hline Especie & Vegetación & $\begin{array}{c}\text { Altitud } \\
\text { (en metros) }\end{array}$ & $\begin{array}{l}\text { Forma } \\
\text { de vida }\end{array}$ \\
\hline Festuca aff. rzedowskiana Alexeev & $\mathrm{BO}$ & 2850 & $\mathrm{HA}$ \\
\hline Lasiacis nigra Davidse & BMM & 2300 & $\mathrm{HP}$ \\
\hline Muhlenbergia confusa (Fourn.) Swallen & LIT & 2400 & $\mathrm{HA}$ \\
\hline Muhlenbergia aff. distans Swallen & ARV & 2800 & HP \\
\hline * Muhlenbergia dumosa Scribn. ex Vasey & & & $\mathrm{HP}$ \\
\hline Muhlenbergia glabrata (HBK.) Kunth & LIT & 2300 & HP \\
\hline Muhlenbergia macrotis (Piper) Hitchc. & BPE & 2050 & $\mathrm{HP}$ \\
\hline Muhlenbergia macroura (HBK.) Hitchc. & PAZ,BPE & $2400-2550$ & $\mathrm{HP}$ \\
\hline Muhlenbergia minutissima (Steud.) Swallen & $\mathrm{PAZ}$ & 2550 & $\mathrm{HA}$ \\
\hline Muhlenbergia pusilla Steud. & $\mathrm{BO}, \mathrm{BP}, \mathrm{ARV}$ & $2050-2850$ & $\mathrm{HA}$ \\
\hline Muhlenbergia ramulosa (HBK.) Swallen & ARV & 1900 & $\mathrm{HA}$ \\
\hline Muhlenbergia virletii (E. Fourn.) Soderstr. & PAS,BPE & $2500-2650$ & $\mathrm{HP}$ \\
\hline Muhlenbergia sp. & ARV & 2700 & $\mathrm{HP}$ \\
\hline Oplismenus burmannii (Retz.) P. Beauv. & BMM & 1900 & $\mathrm{HP}$ \\
\hline Oplismenus compositus (L.) P. Beauv. & BMM & $1900-2000$ & $\mathrm{HP}$ \\
\hline Panicum albomaculatum Scribn. & BMM & 1900 & $\mathrm{HP}$ \\
\hline Panicum bulbosum HBK. & $\mathrm{BP}$ & 2400 & $\mathrm{HP}$ \\
\hline * Panicum decolorans HBK. & $\mathrm{BP}$ & & $\mathrm{HA}$ \\
\hline Panicum hallii Vasey & $\mathrm{BP}$ & 2400 & $\mathrm{HP}$ \\
\hline Paspalum prostratum Scribn. \& Merr. & RUD & 2550 & HP \\
\hline * Paspalum squamulatum Fourn. & $\mathrm{BP}$ & & $\mathrm{HP}$ \\
\hline Phalaris canariensis $\mathrm{L}$. & BMM & 2400 & $\mathrm{HA}$ \\
\hline * Piptochaetium fimbriatum (HBK.) Hitchc. & BPE & & $\mathrm{HP}$ \\
\hline Piptochaetium virescens (HBK.) Parodi & BOP,BPE,BP & $2450-3100$ & $\mathrm{HP}$ \\
\hline Poa annua L. & BPE,BOP & $2500-2800$ & $\mathrm{HP}$ \\
\hline Schizachyrium sanguineum (Retz.) Alston & $\mathrm{BPE}$ & 2400 & $\mathrm{HP}$ \\
\hline Setaria geniculata (Lam.) P. Beauv. & PAS & 2250 & $\mathrm{HP}$ \\
\hline Setaria grisebachii Fourn. & LIT & 2700 & $\mathrm{HA}$ \\
\hline Sporobolus indicus (L.) R. Br. & ARV & 2150 & $\mathrm{HP}$ \\
\hline Triniochloa stipoides (HBK.) Hitchc. & $\mathrm{BP}$ & 2600 & $\mathrm{HP}$ \\
\hline Trisetum altijugum (Fourn.) Scribn. & BO,ARV & $2800-2850$ & $\mathrm{HP}$ \\
\hline Trisetum deyeuxioides (HBK.) Kunth & $\mathrm{BP}$ & 2400 & $\mathrm{HP}$ \\
\hline Trisetum virletii Fourn. & $\mathrm{BO}, \mathrm{BPO}, \mathrm{BP}$ & $2550-3200$ & SB \\
\hline Vulpia myuros (L.) C. C. Gmel. & PAS,BPE,RUD,ARV & $2150-2600$ & $\mathrm{HA}$ \\
\hline Zeugites americana Willd. var. americana & $\mathrm{ARV}, \mathrm{BMM}$ & $2150-2500$ & $\mathrm{HP}$ \\
\hline \multicolumn{4}{|l|}{ Zeugites americana var. pringlei (Scribn.) } \\
\hline McVaugh & BMM & 2450 & $\mathrm{HP}$ \\
\hline \multicolumn{4}{|l|}{ GUTTIFERAE } \\
\hline Clusia salvinii Donn. Sm. & BMM & 2000 & $A B$ \\
\hline * Hypericum aff. pauciflorum HBK. & & & $\mathrm{HA}$ \\
\hline Hypericum philonotis Cham. \& Schltdl. & LIT & 2300 & $\mathrm{HA}$ \\
\hline \multicolumn{4}{|l|}{ HYDROPHYLLACEAE } \\
\hline Nama dichotomum (Ruiz \& Pav.) Choisy & BMM & 2500 & $\mathrm{HA}$ \\
\hline
\end{tabular}


Apéndice. Continuación.

\begin{tabular}{|c|c|c|c|}
\hline Especie & Vegetación & $\begin{array}{c}\text { Altitud } \\
\text { (en metros) }\end{array}$ & $\begin{array}{l}\text { Forma } \\
\text { de vida }\end{array}$ \\
\hline $\begin{array}{l}\text { Phacelia platycarpa (Cav.) Spreng. } \\
\text { Wigandia urens (Ruiz \& Pav.) HBK. }\end{array}$ & $\begin{array}{l}\text { ARV,BPE } \\
\text { MS }\end{array}$ & $\begin{array}{l}2150-2800 \\
2400\end{array}$ & $\begin{array}{l}\mathrm{HA} \\
\mathrm{AB}\end{array}$ \\
\hline \multicolumn{4}{|l|}{ IRIDACEAE } \\
\hline $\begin{array}{l}\text { Sisyrinchium angustifolium Mill. } \\
\text { Sisyrinchium cernuum (E. P. Bicknell) }\end{array}$ & $\mathrm{BP}, \mathrm{LIT}$ & & $\mathrm{HA}$ \\
\hline Kearney & ARV & $2400-2800$ & $\mathrm{HA}$ \\
\hline Sisyrinchium convolutum Nocca & BC & 2550 & HP \\
\hline Sisyrinchium palmeri Greenm. & $\mathrm{BC}$ & 3200 & $\mathrm{HP}$ \\
\hline * Sisyrinchium schaffneri S. Watson & $\mathrm{BP}, \mathrm{BE}, \mathrm{MS}$ & & $\mathrm{HP}$ \\
\hline Sisyrinchium tolucense Peyr. & $\mathrm{BP}$ & 2500 & $\mathrm{HP}$ \\
\hline Tigridia alpestris ssp. obtusa Molseed & BMM & 2600 & $\mathrm{HP}$ \\
\hline \multicolumn{4}{|l|}{ JUNCACEAE } \\
\hline Luzula gigantea Desv. & $\mathrm{BC}$ & 2800 & $\mathrm{HP}$ \\
\hline Luzula racemosa Desv. & $\mathrm{BC}$ & 2800 & $\mathrm{HP}$ \\
\hline \multicolumn{4}{|l|}{ LABIATAE } \\
\hline Hyptis mutabilis (Rich.) Briq. & BMM & 1900 & HP \\
\hline Lepechinia caulescens (Ortega) Epling & ARV & 2150 & $\mathrm{HP}$ \\
\hline Prunella vulgaris $\mathrm{L}$. & $\mathrm{BC}$ & 2900 & $\mathrm{HP}$ \\
\hline Salvia albo-caerulea Linden & BMM & 2000 & $A B$ \\
\hline Salvia clinopodioides HBK. & $\mathrm{BPE}$ & 2500 & $\mathrm{HP}$ \\
\hline Salvia elegans Vahl & BP,BPE,BPO,BOP & $2150-3200$ & HP \\
\hline Salvia fulgens Cav. & BMM & 2400 & $A B$ \\
\hline Salvia gesneriflora Lindl. & $\mathrm{BP}$ & 2150 & $A B$ \\
\hline Salvia gracilis Benth. & $\mathrm{BO}, \mathrm{BP}$ & & $\mathrm{HP}$ \\
\hline Salvia iodantha Fernald & BMM,BPE,BP,BOP & $2500-3100$ & $\mathrm{HP}$ \\
\hline Salvia laevis Benth. & $\mathrm{BO}, \mathrm{BP}$ & & HP \\
\hline Salvia lavanduloides HBK. & $\mathrm{BO}, \mathrm{BPO}, \mathrm{BP}$ & $2600-2950$ & $\mathrm{HP}$ \\
\hline Salvia melissodora Lag. & $\mathrm{BP}$ & & $\mathrm{HP}$ \\
\hline Salvia mexicana L. var. mexicana & $\mathrm{BO}$ & 2700 & $A B$ \\
\hline Salvia mexicana var. minor Benth. & $\mathrm{BO}, \mathrm{BPE}, \mathrm{BP}$ & $2600-2700$ & $A B$ \\
\hline Salvia mocinoi Benth. & BMM & 1900 & $A B$ \\
\hline Salvia polystachya Ortega & BMM & 1900 & $A B$ \\
\hline * Salvia purpurea Cav. & $\mathrm{BP}, \mathrm{BE}$ & & $\mathrm{HP}$ \\
\hline * Salvia sanctae-Iuciae Seem. & & & $A B$ \\
\hline Salvia thyrsiflora Benth. & BMM & $1900-2300$ & HP \\
\hline Salvia tiliifolia Vahl & ARV & 2150 & $\mathrm{HA}$ \\
\hline Satureja macrostema (Benth.) Briq. & $\mathrm{BPE}, \mathrm{BPO}, \mathrm{BO}$ & $2450-2900$ & $A B$ \\
\hline * Scutellaria hintoniana Epling & $\mathrm{BO}, \mathrm{MS}$ & & $\mathrm{HP}$ \\
\hline Stachys coccinea Jacq. & $\mathrm{BC}$ & 2900 & HP \\
\hline Stachys eriantha Benth. & BMM & 2600 & $\mathrm{HA}$ \\
\hline Stachys guatemalensis Epling & $\mathrm{BO}$ & 3000 & $\mathrm{HA}$ \\
\hline Stachys sanchezii Rzed. \& García Zuñiga & BMM & $1900-2350$ & $\mathrm{HP}$ \\
\hline
\end{tabular}


Acta Botanica Mexicana (2000), 52: 5-41

Apéndice. Continuación.

\begin{tabular}{l} 
Especie \\
\hline $\begin{array}{c}\text { Stachys aff. sanchezii Rzed. \& García } \\
\text { Zuñiga }\end{array}$
\end{tabular}

Vegetación

Altitud

Forma

(en metros) de vida

LAURACEAE

Persea americana Mill.

Persea longipes (Schltdl.) Meissn.

LEGUMINOSAE

Acacia angustissima (Mill.) Kuntze var. angustissima

*Acacia farnesiana (L.) Willd.

Astragalus guatemalensis Hemsl.

* Astragalus lyonnetii Barneby

Brongniartia sp.

Calliandra grandiflora (L'Hér.) Benth.

Cologania broussonetii (Balb.) DC.

Cologania rufescens Rose

Crotalaria longirostrata Hook. \& Arn.

Crotalaria mollicula HBK.

* Crotalaria pumila Ortega

Crotalaria rotundifolia (Walter) J. F. Gmel. var. rotundifolia

BMM

ARV

BP

BPE

$B P, B E$

RUD

BP

BMM

BPE

MS,PAS

BPE

BP,MS

RUD

$\mathrm{BP}$

BPE

BPE

$\mathrm{BP}$

RUD

BMM

BP,BE,BMM

BE,BMM

MS,PAZ

BO,BMM,MS

BPE

BPE

Desmodium aparines (Link) DC.

Desmodium bellum (S. F. Blake) B. G.

Schub.

Desmodium densiflorum Hemsl.

Desmodium neomexicanum A. Gray

* Desmodium plicatum Schltdl. \& Cham. Desmodium sumichrastii (Schindl.) Standl. Desmodium uncinatum (Jacq.) DC.

Eysenhardtia polystachya (Ortega) Sarg.

* Lathyrus parvifolius S. Watson

Leucaena esculenta (DC.) Benth.

* Lotus angustifolius (G. Don) Sessé \& Moc. Lotus repens (G. Don) Standl. \& Steyerm. Lupinus campestris Schltdl. \& Cham.

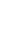

BPE,BP
BMM
BPE
BPE,BP,MS
BMM
BP
BMM
BP,BPE
BPE,MS
BP,BOP

2350

HP

2150

2150

AR

AR

$2400-2550$
2300
2000
2100
$2300-2400$
2400
2300
2300

2400
2400
2300
2500
2000

$A B$

$A B$

$\mathrm{HP}$

$\mathrm{HP}$

$A B$

$A B$

TR

TR

HP

HP

$\mathrm{HA}$

HP

HP

$\mathrm{HA}$

HP

$A B$

HA

HA

2450-2550

$\mathrm{HA}$

$A B$

HP

2400

TR

AB

2150-2650

2550

$A B$

$\mathrm{HA}$

$A B$

2400-2500

2400-2600

2400

$A B$

$\mathrm{HP}$

$A B$

HP

1900

AR

HP

2400-2650

2750-3100

HP

HP 
Apéndice. Continuación.

Especie

\section{Vegetación}

Lupinus elegans HBK.

Lupinus exaltatus Zucc.

* Lupinus marshallianus Sweet

Lupinus mexicanus Cerv. ex Lag.

* Lupinus montanus HBK.

* Lupinus persistens Rose Lupinus reflexus Rose Lupinus splendens Rose

* Lupinus stipulatus J. Agardh

Macroptilium gibbosifolium (Ortega) A. Delgado

Marina grammadenia Barneby

Marina nutans (Cav.) Barneby

Marina scopa Barneby

Mimosa albida Humb. \& Bonpl. ex Willd.

Phaseolus acutifolius var. latifolius G. F. Freeman

Phaseolus coccineus L.

Phaseolus lunatus L.

* Phaseolus micranthus Hook. \& Arn. var. micranthus

* Phaseolus pedicellatus Benth.

Phaseolus vulgaris $\mathrm{L}$.

Senna multiglandulosa (Jacq.) H. S. Irwin \& Barneby

Trifolium amabile HBK.

Trifolium goniocarpum Lojac.

* Vicia humilis HBK.

* Vicia pulchella HBK. ssp. pulchella

* Vicia pulchella ssp. mexicana (Hemsl.)

C. R. Gunn

\section{LENTIBULARIACEAE}

Pinguicula moranensis HBK.

Pinguicula oblongiloba DC.

\section{LILIACEAE}

Echeandia durangensis (Greenm.) Cruden

* Echeandia flavescens (Schult. \& Schult.

$$
\text { f.) Cruden }
$$

Echeandia mexicana Cruden

\section{LINACEAE}

Linum orizabae Planch.
Altitud

(en metros)

BPE,BP,BMM,BO
PAZ,MS
BPE
BO,BP,BPE,MS
BP
BP
BP
BC
BPO
BO,BPE

ARV

BMM

BMM

RUD

BMM

BMM

BMM

BPE

2400-3200

Forma

de vida

BP

BP,BE,BPE,MS

BMM

2450

2150

3250

3100

AB

HP

HP

HP

HP

HP

$A B$

HP

HP

2150

2000

1900

2300

2000

HP

$A B$

HP

$\mathrm{HA}$

$A B$

2000

2000

TR

2350

TR

TR

BMM

MS,PAS

BP

BP

BP

HA

HP

2000

TR

2500

2300-2600

$A B$

2600

HP

HP

TR

TR

TR

BMM

2450

2650

HP

HP

BC

2850

HP

BP,BE,BMM

BPE,BMM

$1900-2400$

HP

HP

BPE

2400

HP 
Acta Botanica Mexicana (2000), 52: 5-41

Apéndice. Continuación.

Especie
Altitud (en metros)

Forma de vida

LOGANIACEAE

Buddleia cordata HBK. ssp. cordata

Buddleia parviflora HBK.

* Buddleia sessiliflora HBK.

LORANTHACEAE

Arceuthobium globosum Hawksw. \& Wiens ssp. globosum

* Arceuthobium globosum ssp. grandicaule Hawksw. \& Wiens

Cladocolea microphylla (HBK.) Kuijt

Cladocolea pedicellata Kuijt

Phoradendron falcatum (Schltdl. \& Cham.) Trel.

* Phoradendron longifolium Eichler ex Trel.

* Psittacanthus calyculatus (DC.) G. Don Psittacanthus macrantherus Eichler

* Struthanthus microphyllus (HBK.) G. Don

* Struthanthus venetus (HBK.) Blume

LYTHRACEAE

* Cuphea aequipetala Cav. Cuphea bustamanta Lex. Cuphea hookeriana Walp.

* Cuphea jorullensis HBK.

MALVACEAE

* Anoda cristata (L.) Schltdl. Kearnemalvastrum subtriflorum (Lag.)

D. M. Bates

Malvaviscus arboreus Cav.

Neobrittonia acerifolia (G. Don) Hochr.

Phymosia rosea (DC.) Kearney

Sida haenkeana C. Presl

MELASTOMATACEAE

Heterocentron mexicanum Hook. \& Arn.

Leandra subseriata (Naudin) Cogn.

MYRSINACEAE

Parathesis macronema Bullock

Parathesis melanosticta (Schltdl.) Hemsl.

Parathesis sp.

Rapanea jurgensenii Mez

MYRTACEAE

Myrcianthes fragrans (Sw.) McVaugh
BPE

BPE

$B E$

BPO

$\mathrm{BC}$

$\mathrm{BC}$

$\mathrm{BC}$

BP

BC

BP

BMM

BMM

BP

ARV

BMM

BMM

BMM

BMM

BMM

BMM

BMM

BP

BMM

BP

BP
2400

2450-2600

AR

$A B$

$A B$

2700

PA

PA

PA

PA

2700

2700

PA

PA

PA

PA

PA

PA

HP

2300-2450

2000

$\mathrm{HA}$

HP

HP

HP

2900

SB

1900-2000 SB

2050

2000

2500

$A B$

$A B$

SB

1900

2000

HA

$A B$

1900-2000

$A B$

2150

2550

2150

AR

AR

$A B$

2150

AR 
Apéndice. Continuación.

\begin{tabular}{|c|c|c|c|}
\hline Especie & Vegetación & $\begin{array}{c}\text { Altitud } \\
\text { (en metros) }\end{array}$ & $\begin{array}{l}\text { Forma } \\
\text { de vida }\end{array}$ \\
\hline
\end{tabular}

\section{ONAGRACEAE}

Epilobium ciliatum Raf.

Fuchsia arborescens Sims

Fuchsia fulgens DC.

Fuchsia microphylla HBK.

Fuchsia obconica Breedlove

Fuchsia parviflora (Zucc.) Hemsl.

Fuchsia thymifolia HBK.

Lopezia miniata Lag. ex DC.

Lopezia racemosa Cav.

Lopezia trichota Schltdl.

Oenothera pubescens Willd. ex Spreng.

Oenothera purpusii Munz

\begin{tabular}{|c|c|c|}
\hline BMM & 2500 & $\mathrm{HP}$ \\
\hline BMM,BP & $1900-2150$ & $A B$ \\
\hline $\mathrm{BP}, \mathrm{BMM}$ & 2500 & $A B$ \\
\hline BO,BMM,BOP,BPO,BPE & $2450-3200$ & $A B$ \\
\hline BOP & $2400-2550$ & $A B$ \\
\hline BMM,BPE,MS & $1900-2650$ & $A B$ \\
\hline BMM,BPE & $1900-2400$ & $A B$ \\
\hline BMM & 2000 & $\mathrm{HA}$ \\
\hline ARV,BPE,BP & $2150-2600$ & HA \\
\hline $\mathrm{BC}$ & 3200 & HP \\
\hline ARV,LIT,BP,PAZ,BPE & $2150-3200$ & $\mathrm{HP}$ \\
\hline $\mathrm{BPO}$ & 2750 & $\mathrm{HA}$ \\
\hline LIT & & HP \\
\hline BPE & 2400 & SA \\
\hline BP & & SA \\
\hline BO & & EP \\
\hline BO & & EP \\
\hline BPE & 2400 & $\mathrm{HP}$ \\
\hline BC & 2600 & $\mathrm{HP}$ \\
\hline BC & 2500 & $\mathrm{HP}$ \\
\hline BPE & 2500 & $\mathrm{HP}$ \\
\hline BPE & 2500 & $\mathrm{HP}$ \\
\hline & & HP \\
\hline $\mathrm{BC}$ & 3000 & $\mathrm{HP}$ \\
\hline BC & 3000 & $\mathrm{HP}$ \\
\hline BPE & 2500 & $\mathrm{HA}$ \\
\hline BPE,LIT,BMM & 2400 & HP \\
\hline BC & 2500 & $\mathrm{HP}$ \\
\hline BPE,BPO & $2450-2750$ & $\mathrm{HP}$ \\
\hline LIT & 2400 & $\mathrm{HP}$ \\
\hline BO & & $\mathrm{HP}$ \\
\hline BO & 3000 & $\mathrm{HP}$ \\
\hline$B C$ & 2550 & $\mathrm{HP}$ \\
\hline LIT & 2700 & $\mathrm{HP}$ \\
\hline ARV & 2150 & HP \\
\hline BP,BE & & $\mathrm{HP}$ \\
\hline BC & 2650 & $\mathrm{HP}$ \\
\hline
\end{tabular}

ORCHIDACEAE

* Bletia rosea A.Rich. \& Galeotti

Corallorrhiza involuta Greenm.

* Corallorrhiza maculata Raf.

* Epidendrum anisatum Lex.

* Epidendrum cusii Hágsater

Govenia liliacea (Lex.) Lindl.

Govenia purpusii Schltr. vel aff.

Govenia superba (Lex.) Lindl. ex Lodd.

Habenaria guadalajarana S. Watson

Habenaria novemfida Lindl.

Habenaria sp.

Liparis draculoides E. W. Greenw.

Malaxis ehrenbergii (Rchb. f.) Kuntze

Malaxis unifolia Michx.

Ponthieva sp.

Rhynchostele cervantesii (Lex.) Soto Arenas \& Salazar

Spiranthes eriophora B. L. Rob. \&

Greenm.

Spiranthes graminea Lindl.

* Spiranthes hyemalis A. Rich. \& Galeotti Spiranthes rubrocallosa B. L. Rob. \& Greenm.

\section{OXALIDACEAE}

Oxalis alpina (Rose) Knuth

Oxalis albicans HBK.

Oxalis corniculata L.

* Oxalis hernandesii DC.

Oxalis latifolia HBK. 
Acta Botanica Mexicana (2000), 52: 5-41

Apéndice. Continuación.

\begin{tabular}{|c|c|c|c|}
\hline Especie & Vegetación & $\begin{array}{c}\text { Altitud } \\
\text { (en metros) }\end{array}$ & $\begin{array}{l}\text { Forma } \\
\text { de vida }\end{array}$ \\
\hline \multicolumn{4}{|l|}{ PASSIFLORACEAE } \\
\hline Passiflora biflora Lam. & BMM & 2400 & TR \\
\hline Passiflora coriacea Juss. & BMM & 1900 & TR \\
\hline Passiflora filipes Benth. & BMM & 2300 & TR \\
\hline Passiflora sp. & BMM & 2000 & TR \\
\hline \multicolumn{4}{|l|}{ PHYTOLACCACEAE } \\
\hline Phytolacca icosandra L. & $\mathrm{BMM}, \mathrm{ARV}$ & $1900-2150$ & $A B$ \\
\hline \multicolumn{4}{|l|}{ PIPERACEAE } \\
\hline Peperomia callocata Trel. & BMM & $1900-2400$ & $\mathrm{HA}$ \\
\hline Peperomia campylotropa A. W. Hill & $\mathrm{BP}, \mathrm{BMM}$ & $2400-2550$ & HP \\
\hline Peperomia galioides HBK. & BPE,BP,LIT,BPO & $2450-2700$ & EP \\
\hline Peperomia hintonii Yunck. & BMM & 2500 & $\mathrm{HA}$ \\
\hline Peperomia hispidula (Sw.) A. Dietr. & BMM,BPO & $2450-2800$ & EP \\
\hline Peperomia quadrifolia (L.) HBK. & $\mathrm{BC}$ & 2500 & EP \\
\hline \multicolumn{4}{|l|}{ Peperomia tetraphylla (G. Forst.) Hook. \& } \\
\hline Arn. & BMM & 1900 & EP \\
\hline Piper diandrum DC. & BMM & 2300 & $A B$ \\
\hline Piper sp. & BMM & 2000 & $A B$ \\
\hline \multicolumn{4}{|l|}{ PLANTAGINACEAE } \\
\hline Plantago australis Lam. ssp. australis & BPO & 2900 & HP \\
\hline * Plantago australis ssp. hirtella (HBK.) Rahn & $\mathrm{BO}, \mathrm{BP}, \mathrm{BMM}$ & & HP \\
\hline Plantago linearis var. mexicana (Link) Pilg. & RUD & 2400 & HP \\
\hline Plantago linearis var. villosa Pilg. & BO & 2850 & HP \\
\hline Plantago major L. & RUD & 2850 & HP \\
\hline \multicolumn{4}{|l|}{ POLEMONIACEAE } \\
\hline * Loeselia mexicana (Lam.) Brand & & & SB \\
\hline \multicolumn{4}{|l|}{ POLYGALACEAE } \\
\hline Monnina ciliolata DC. & BPE & $2400-2550$ & $A B$ \\
\hline Monnina xalapensis HBK. & BPE & 2300 & $A B$ \\
\hline \multicolumn{4}{|l|}{ POLYGONACEAE } \\
\hline Polygonum hydropiperoides Michx. & PAZ & 2650 & HP \\
\hline Polygonum punctatum Elliott & BMM & 2500 & HP \\
\hline Rumex conglomeratus Murray & PAZ & 2650 & HP \\
\hline Rumex obtusifolius L. & ARV & 2300 & HP \\
\hline \multicolumn{4}{|l|}{ PONTEDERIACEAE } \\
\hline Heteranthera limosa (Sw.) Willd. & SAC & 2900 & $A C$ \\
\hline \multicolumn{4}{|l|}{ PORTULACACEAE } \\
\hline Claytonia perfoliata Donn ex Willd. & BC & 2900 & $\mathrm{HA}$ \\
\hline
\end{tabular}


Apéndice. Continuación.

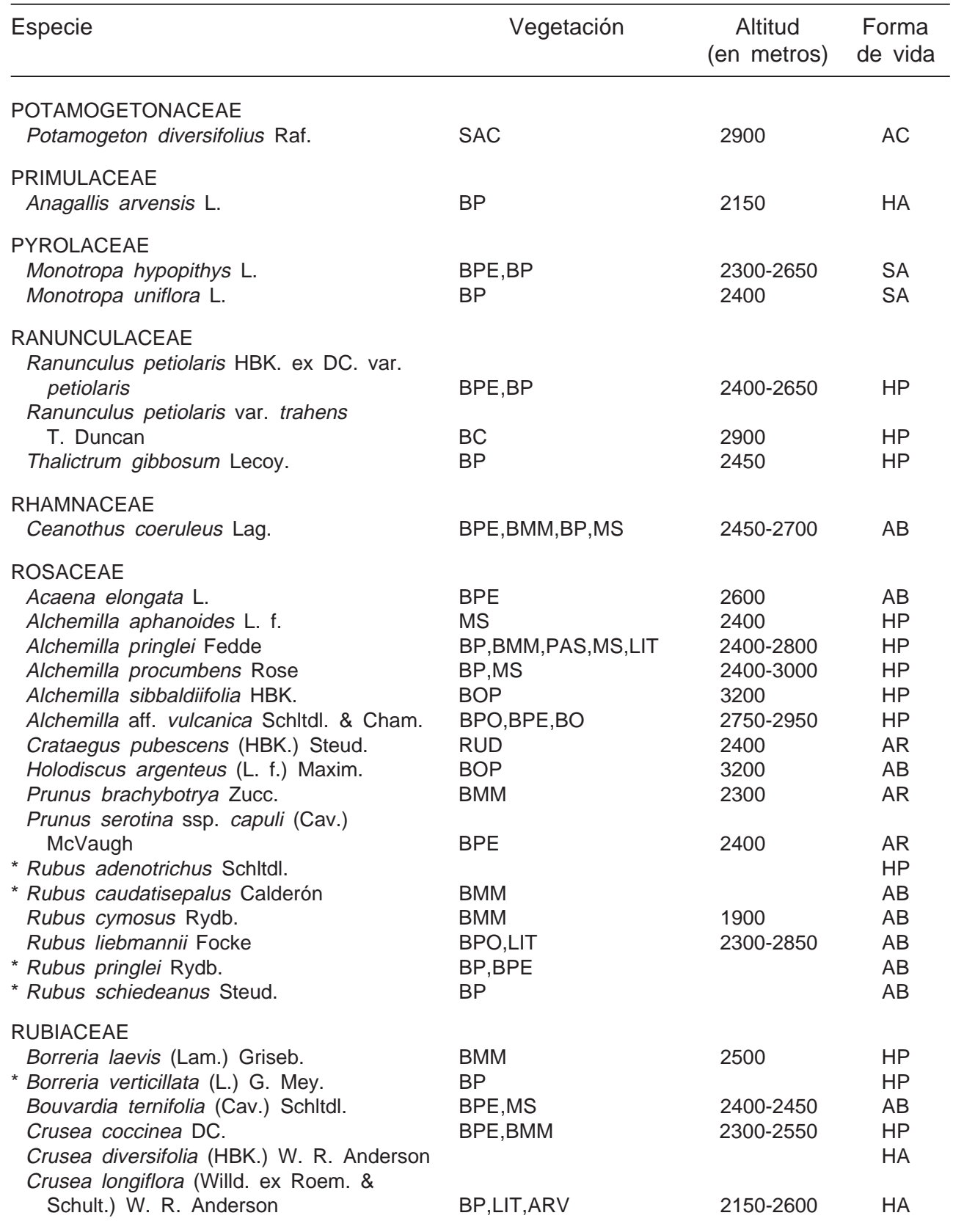


Acta Botanica Mexicana (2000), 52: 5-41

Apéndice. Continuación.

Especie
Didymaea alsinoides (Schltdl. \& Cham.)

Standl.

Didymaea floribunda Rzed.

Didymaea sp.

Galium aschenbornii Schauer

Galium mexicanum HBK.

Hedyotis pygmaea Roem. \& Schult.

Psychotria sp.

Vegetación

Altitud

Forma

(en metros) de vida

SABIACEAE

Meliosma dentata (Liebm.) Urb.

BPE,BP,BPO,BO
BMM
BO
BP,BPO
BPE,BOP,BPO,BP
LIT
BMM

2400-3000

HP

2450

2800

TR

2600-2800 TR

2650-3100 TR

$2400 \quad H P$

BMM

2300

$A B$

BMM

2400

AR

SALICACEAE

Salix bonplandiana HBK.

BMM

1900

Salix paradoxa HBK.

$\mathrm{BPE}, \mathrm{BP}, \mathrm{BPO}, \mathrm{BO}$

2400-3200

AR

SAXIFRAGACEAE

Heuchera orizabensis Hemsl.

BPE

$2550-2850$

HP

SCROPHULARIACEAE

Bacopa procumbens (Mill.) Greenm.

Buchnera obliqua Benth.

Castilleja arvensis Cham. \& Schltdl.

Castilleja gracilis Benth.

Castilleja lithospermoides HBK.

* Castilleja moranensis HBK.

Castilleja scorzonerifolia HBK.

Castilleja tenuiflora Benth.

Castilleja tenuifolia M. Martens \& Galeotti

Lamourouxia multifida HBK.

Lamourouxia xalapensis HBK.

Penstemon apateticus Straw

Penstemon campanulatus (Cav.) Willd.

Russelia multiflora Sims

Russelia sp.

Sibthorpia repens (Mutis ex L. f.) Kuntze

* Verbascum virgatum Stokes ex With.

LIT

LIT

BPO,BMM

BMM

$\mathrm{BO}$

$\mathrm{BO}, \mathrm{BP}$

$\mathrm{BC}$

BPE,BPO,ARV

BPE,ARV

BP

BPE

BPE

BOP

BMM

BMM

BMM,BPE

BE

HP

HP

1900-2800 HA

1900

HA

2850

HP

HA

2750

2150-2850

HP

2150-2400

HP

2600

2900

2900

3200

1900

1900

2400-2450

$\mathrm{HP}$

HP

AB

$\mathrm{HP}$

$\mathrm{HP}$

$A B$

$A B$

$\mathrm{HP}$

$\mathrm{HA}$

SMILACACEAE

Smilax moranensis M. Martens \& Galeotti Smilax pringlei Greenm.

BP,BPE

BMM

BMM,BE

BPE,BMM

BPO,BPE,BP

BMM
2450-2700

2450-2550

TR

TR

2150-2750

$A B$

2450-2650

2450-2750

2300
$A B$

$A B$

$A B$ 
Apéndice. Continuación.

\begin{tabular}{|c|c|c|c|}
\hline Especie & Vegetación & $\begin{array}{c}\text { Altitud } \\
\text { (en metros) }\end{array}$ & $\begin{array}{l}\text { Forma } \\
\text { de vida }\end{array}$ \\
\hline $\begin{array}{l}\text { Jaltomata procumbens (Cav.) J. L. Gentry } \\
\text { Physalis costomatl Moc. \& Sessé ex }\end{array}$ & BMM,BO,BOP,PAZ,ARV & $2150-3200$ & $\mathrm{HA}$ \\
\hline Dunal & $\mathrm{BC}$ & 2500 & $A B$ \\
\hline Physalis orizabae Dunal & $\mathrm{BC}$ & 2500 & HP \\
\hline Physalis sordida Fernald & $\mathrm{BP}$ & 2400 & $\mathrm{HA}$ \\
\hline Physalis stapelioides (Regel) Bitter & ARV & 2150 & HP \\
\hline * Physalis sulphurea (Fernald) Waterf. & $\mathrm{BO}, \mathrm{BP}, \mathrm{BE}, \mathrm{BPE}$ & & $\mathrm{HA}$ \\
\hline Physalis volubilis Waterf. & BPE,BPO,BP & $2500-3000$ & HP \\
\hline Solanum americanum Mill. & ARV & 2150 & HP \\
\hline $\begin{array}{l}\text { Solanum appendiculatum Humb. \& Bonpl. } \\
\text { ex Dunal }\end{array}$ & RUD,BPO,BE & $2700-2800$ & TR \\
\hline Solanum cervantesii Lag. & BMM & 2300 & $A B$ \\
\hline Solanum aff. dulcamaroides Dunal & BMM,BPE & $2400-2500$ & TR \\
\hline Solanum lanceolatum Cav. & BMM,ARV & 2500 & $A B$ \\
\hline Solanum mozinianum Dunal & BMM & 2500 & HP \\
\hline Solanum nigrescens M. Martens \& Galeotti & PAS & 2400 & HP \\
\hline Solanum nudum Dunal & BMM & $2300-2450$ & $A B$ \\
\hline Solanum torvum Sw. & BMM & 2000 & $A B$ \\
\hline Solanum verrucosum Schltdl. & $\mathrm{BC}$ & 2500 & HP \\
\hline \multicolumn{4}{|l|}{ STYRACACEAE } \\
\hline $\begin{array}{l}\text { Styrax argenteus C. Presl ssp. argenteus } \\
\text { Styrax argenteus var. ramirezii (Greenm.) }\end{array}$ & BP & 2150 & AR \\
\hline Gonsoulin & BMM & 1900 & AR \\
\hline \multicolumn{4}{|l|}{ SYMPLOCACEAE } \\
\hline Symplocos citrea Lex. & $\mathrm{BP}, \mathrm{BPO}$ & $2600-2800$ & AR \\
\hline \multicolumn{4}{|l|}{ THEACEAE } \\
\hline Cleyera integrifolia (Benth.) Choisy & BMM & $2300-2450$ & AR \\
\hline Ternstroemia pringlei (Rose) Standl. & BPE & 2450 & AR \\
\hline \multicolumn{4}{|l|}{ TILIACEAE } \\
\hline Tilia mexicana Schltdl. & BPE & 2650 & AR \\
\hline * Tilia occidentalis Rose & BPE & 2650 & AR \\
\hline * Triumfetta brevipes S. Watson & $\mathrm{BP}$ & & $A B$ \\
\hline * Triumfetta galeottiana Turcz. & BO & & $A B$ \\
\hline \multicolumn{4}{|l|}{ ULMACEAE } \\
\hline Trema micrantha (L.) Blume & BMM & 1900 & AR \\
\hline \multicolumn{4}{|l|}{ UMBELLIFERAE } \\
\hline $\begin{array}{l}\text { Apium leptophyllum (Pers.) F. Muell. } \\
\text { Arracacia aegopodioides (HBK.) J. M. }\end{array}$ & ARV & 2150 & HP \\
\hline $\begin{array}{l}\text { Coult. \& Rose } \\
\text { Arracacia atropurpurea (Lehm.) Benth. \& }\end{array}$ & $\mathrm{BC}$ & 2500 & HP \\
\hline Hook. & BMM,BPE,BPO & $2300-2950$ & HP \\
\hline
\end{tabular}


Acta Botanica Mexicana (2000), 52: 5-41

Apéndice. Continuación.

\begin{tabular}{|c|c|c|c|}
\hline Especie & Vegetación & $\begin{array}{c}\text { Altitud } \\
\text { (en metros) }\end{array}$ & $\begin{array}{l}\text { Forma } \\
\text { de vida }\end{array}$ \\
\hline \multicolumn{4}{|l|}{ *Arracacia tolucensis var. multifida (S. } \\
\hline Daucus montanus Humb. \& Bonpl. & BMM & 2300 & $\mathrm{HA}$ \\
\hline \multicolumn{4}{|l|}{ Donnellsmithia mexicana (B. L. Rob.) } \\
\hline Mathias \& Constance & BMM & $1900-2000$ & HP \\
\hline Eryingium alternatum J. M. Coult. \& Rose & BPE,BPO & $2650-3100$ & $\mathrm{HP}$ \\
\hline Eryngium carlinae F. Delaroche & BPE & 2600 & $\mathrm{HP}$ \\
\hline Eryngium longifolium Cav. & BPO,BPE,BP & $2600-2700$ & HP \\
\hline Eryngium mexiae Constance & $\mathrm{BC}$ & 2600 & $\mathrm{HP}$ \\
\hline Rhodosciadium tolucense (HBK.) Mathias & $\mathrm{BC}$ & 2550 & $\mathrm{HP}$ \\
\hline \multicolumn{4}{|l|}{ URTICACEAE } \\
\hline Parietaria pensylvanica Muhl. & $\mathrm{BC}$ & 2500 & $\mathrm{HA}$ \\
\hline Phenax hirtus (Sw.) Wedd. & BMM & 2550 & $\mathrm{HP}$ \\
\hline Urera caracasana (Jacq.) Griseb. & BMM & 2300 & AR \\
\hline Urtica mexicana Liebm. & BMM & 2300 & $\mathrm{HP}$ \\
\hline Urtica urens L. & $\mathrm{BC}$ & 2500 & $\mathrm{HA}$ \\
\hline \multicolumn{4}{|l|}{ VALERIANACEAE } \\
\hline Valeriana clematitis HBK. & $\mathrm{BC}$ & 2600 & TR \\
\hline Valeriana robertianifolia Briq. & LIT & & $\mathrm{HA}$ \\
\hline Valeriana sorbifolia HBK. & $\mathrm{BMM}, \mathrm{BPO}$ & $1900-2800$ & $\mathrm{HA}$ \\
\hline Valeriana urticifolia HBK. & BPE & $2400-2600$ & $\mathrm{HA}$ \\
\hline \multicolumn{4}{|l|}{ VERBENACEAE } \\
\hline Citharexylum affine G. Don. & BMM & 1900 & AR \\
\hline Verbena bipinnatifida Nutt. & LIT & 2700 & $\mathrm{HP}$ \\
\hline Verbena carolina L. & BMM,ARV & $2150-2550$ & $\mathrm{HP}$ \\
\hline \multicolumn{4}{|l|}{ VIOLACEAE } \\
\hline Viola grahamii Benth. & BPE & 2500 & $\mathrm{HP}$ \\
\hline \multicolumn{4}{|l|}{ VITACEAE } \\
\hline Vitis bourgaeana Planch. & BMM & 1900 & TR \\
\hline
\end{tabular}

Vegetación:

BMM bosque mesófilo de montaña
BC bosque de coníferas
BP bosque de pino
BPO bosque de pino-oyamel
BOP bosque de oyamel-pino
BO bosque de oyamel

$\begin{array}{ll}\text { BPE } & \text { bosque de pino-encino } \\ \text { BE } & \text { bosque de encino } \\ \text { PAZ } & \text { pastizal } \\ \text { SAC } & \text { subacuática } \\ \text { MS } & \text { matorral secundario } \\ \text { ARV } & \text { arvenses }\end{array}$

BPE bosque de pino-encino

PAZ pastizal

SAC subacuática

ARV arvenses 
Medina et al.: Estudio Florístico de Nuevo San Juan Parangaricutiro, Michoacán, México

LIT litoserie

PAS pastizal inducido

Forma de vida:

$\begin{array}{ll}\text { AR } & \text { árbol } \\ \text { AB } & \text { arbusto } \\ \text { SB } & \text { subarbusto } \\ \text { HP } & \text { herbácea perenne } \\ \text { HA } & \text { herbácea anual }\end{array}$

RUD ruderal

REF reforestación

$\begin{array}{ll}\text { TR } & \text { trepadora } \\ \text { EP } & \text { epífita } \\ \text { PA } & \text { parásita } \\ \text { SA } & \text { saprófita } \\ \text { AC } & \text { acuática }\end{array}$

